\title{
Renormalization of the radiative jet function
}

\author{
Geoffrey T. Bodwin $\odot,^{1, *}$ June-Haak Ee ${ }^{2,3, \dagger}$ Jungil Lee $\odot,{ }^{2, \ddagger}$ and Xiang-Peng Wang $\oplus^{1, \S}$ \\ ${ }^{1}$ High Energy Physics Division, Argonne National Laboratory, Argonne, Illinois 60439, USA \\ ${ }^{2}$ Department of Physics, Korea University, Seoul 02841, Korea \\ ${ }^{3}$ Key Laboratory of Nuclear Physics and Ion-beam Application (MOE) and Institute of Modern Physics, \\ Fudan University, Shanghai 200433, China
}

(Received 17 August 2021; accepted 15 November 2021; published 29 December 2021)

\begin{abstract}
We show how to compute directly the renormalization/evolution of the radiative jet function that appears in the factorization theorems for $B \rightarrow \gamma \ell \nu$ and $H \rightarrow \gamma \gamma$ through a $b$-quark loop. We point out that, in order to avoid double counting of soft contributions, one should use in the factorization theorems a subtracted radiative jet function from which soft contributions have been removed. The soft-contribution subtractions are zero-bin subtractions in the terminology of soft-collinear effective theory. We show that they can be factored from the radiative jet function and that the resulting soft-subtraction function gives rise to a nonlocal renormalization of the subtracted radiative jet function. This is a novel instance in which zero-bin subtractions lead to a nonlocality in the renormalization of a subtracted quantity that is not present in the renormalization of the unsubtracted quantity. We demonstrate the use of our formalism by computing the order- $\alpha_{s}$ evolution kernel for the subtracted radiative jet function. Our result is in agreement with the result that had been inferred previously by making use of the factorization theorem for $B \rightarrow \gamma \ell \nu$, but that had been ascribed to the unsubtracted radiative jet function.
\end{abstract}

DOI: 10.1103/PhysRevD.104.116025

\section{INTRODUCTION}

In the amplitudes for exclusive processes, contributions in which a quark carries a soft momentum appear at subleading power in the ratio of the quark mass to the large momentum transfer in the process. These contributions arise because there is a pinch singularity in the region of soft quark momentum that has a subleading-power dependence [1]. They are associated with endpoint singularities in light-cone amplitudes. [2-8]. ${ }^{1}$ In the language of soft-collinear effective theory (SCET) [13-17], the softquark contributions occur through a process in which a jet function containing collinear quarks and gluons emits a soft quark via a subleading-power interaction. Such a jet function is called a radiative jet function [18-20].

\footnotetext{
*gtb@anl.gov

june_haak_ee@fudan.edu.cn

jungil@korea.ac.kr

\$xiangpeng.wang@anl.gov
}

${ }^{1}$ There are also analyses of corrections to inclusive cross sections at subleading power in the inverse of the large momentum transfer. See, for example Refs. [9-12].

Published by the American Physical Society under the terms of the Creative Commons Attribution 4.0 International license. Further distribution of this work must maintain attribution to the author(s) and the published article's title, journal citation, and DOI. Funded by SCOAP.
A particular radiative jet function, which is the focus of this paper, enters into the factorization theorem for the exclusive $B$-meson decay $B \rightarrow \gamma \ell \nu$ [21] and the factorization theorem for the exclusive decay of the Higgs boson $H \rightarrow \gamma \gamma$ through a $b$-quark loop [22]. ${ }^{2}$ In the remainder of this paper, we will refer to this jet function as the radiative jet function. The renormalization properties of the radiative jet function are an essential ingredient in using these factorization theorems to resum large logarithms of the ratios of $m_{b} / \mu$ or $m_{H} / m_{b}$, where $\mu$ is the factorization scale, $m_{b}$ is the $b$-quark mass, and $m_{H}$ is the Higgs-boson mass.

The radiative jet function has been computed through order $\alpha_{s}$ in Ref. [22], and we have verified this calculation. It has also been computed through order $\alpha_{s}^{2}$ in Ref. [25].

The renormalization-group evolution of the radiative jet function in order $\alpha_{s}$ has been inferred from the factorization theorem for $B \rightarrow \gamma \ell \nu$, the renormalization-group invariance of the physical amplitude for $B \rightarrow \gamma \ell \nu$, and the known renormalization-group evolution kernel of the $B$-meson light-front distribution, which also appears in the factorization theorem $[21] .{ }^{3}$ That analysis has been extended to

\footnotetext{
${ }^{2} \mathrm{~A}$ discussion of the factorization theorem for the decay $B \rightarrow \gamma \ell \nu$ in the context of the method of regions is given in Ref. [23]. Subleading-power corrections to the decay $B \rightarrow \gamma \ell \nu$ are discussed in Ref. [24].

${ }^{3}$ This is an application of what is called the consistency condition for the renormalization-group evolution [21].
} 
order $\alpha_{s}^{2}$ in Ref. [25]. The renormalization of the radiative jet function that is obtained from these analyses is nonlocal in momentum space in that it involves the convolution of a renormalization factor $Z_{J}$ with the unrenormalized radiative jet function, rather than a simple multiplication.

Although the renormalization properties of the radiative jet function have been known indirectly for almost two decades, a method for computing the nonlocal renormalization factor $Z_{J}$ directly from the definition of the radiative jet function has remained elusive. In the words of Ref. [25], "It is an embarrassment that there is no known method in SCET to derive the anomalous dimensions of the jet functions directly from their operator definitions."

In this paper, we present a method to derive the anomalous dimension of the radiative jet function directly from its operator definition. We point out that the radiative jet function contains soft contributions that are already taken into account in the soft functions of the exclusive factorization theorems. These soft contributions must be subtracted from the radiative jet function in order to avoid double counting. Methods for the systematic subtraction of double-counted soft contributions are familiar from the diagrammatic approach to factorization [26,27] and are known in SCET under the name zero-bin subtractions [28]. We call the radiative jet function with the soft contributions subtracted the subtracted radiative jet function. It is the subtracted radiative jet function, rather than the radiative jet function, that should properly appear in the exclusive factorization theorems.

We show that the soft subtractions can be factored from the radiative jet function into a soft-subtraction function by making use of the Grammer-Yennie approximation [29] and the graphical Ward identities that are standard in diagrammatic factorization [30]. The soft-subtraction function gives rise to nonlocal ultraviolet (UV) divergences and accounts for all of the nonlocal contributions in $Z_{J}$.

In dimensional regularization, soft subtractions (zero-bin subtractions) generally result in scaleless integrals that can be interpreted as being proportional to a difference between UV and infrared (IR) poles. The soft subtractions then have the function of converting IR poles to UV poles. In a fixedorder calculation, if one does not distinguish IR poles from UV poles, then the soft subtractions do not affect the result. As we will see, this is also the case for the soft subtractions of the radiative jet function. However, beyond one-loop order, the UV poles of the soft subtractions are proportional to nonlocal convolutions over light-front momenta, and, so, they lead to nonlocal contributions to $Z_{J}$. To the best of our knowledge, this is the first time that a nonlocal renormalization owing to the effect of zero-bin subtractions has been observed.

The remainder of this paper is organized as follows. In Sec. II we present some of the notation and conventions that we use throughout this paper. In Sec. III we give the operator definition of the radiative jet function and show

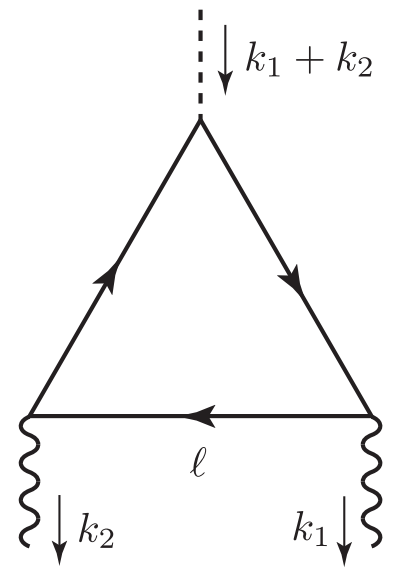

FIG. 1. $H \rightarrow b \bar{b} \rightarrow \gamma \gamma$ at leading order. The dashed line represents the Higgs boson, the solid line represents the $b$ quark, and the wavy lines are the photons.

how to factor the radiative jet function into a convolution of a soft-subtraction function and a subtracted radiative jet function. The renormalization procedure for the subtracted radiative jet function is outlined in Sec. IV. In Sec. V we record the leading-order expressions for the radiative jet function, the soft-subtraction function, the subtracted radiative jet function, and the soft-subtraction renormalization. In Sec. VI we present the application of our method to the renormalization of the subtracted radiative jet function in order $\alpha_{s}$. Our result for the order- $\alpha_{s}$ renormalization-group kernel of the subtracted radiative jet function is in agreement with the result in Ref. [21], although, in that work, the renormalization-group kernel is ascribed to the unsubtracted radiative jet function. In Sec. VII, we argue that soft subtractions generally vanish in dimensional regularization if one does not distinguish between UV and IR divergences, and, hence, do not affect existing fixedorder calculations of the radiative jet function. Finally, we summarize and discuss our results in Sec. VIII.

\section{NOTATION AND CONVENTIONS}

In this section, we establish some of our notation and conventions.

The lowest-order contribution to $H \rightarrow \gamma \gamma$ through a $b$ quark loop is shown in Fig. 1, which establishes our conventions for the momenta of the external particles and the orientation of the internal quark loop.

We adopt dimensional regularization in $D=4-2 \epsilon$ space-time dimensions to regularize the divergences in the loop integrations.

We define two lightlike vectors, $n$ and $\bar{n}$, which satisfy the conditions

$$
n^{2}=0, \quad \bar{n}^{2}=0, \quad n \cdot \bar{n}=2 .
$$

Any four-vector $\ell$ can be decomposed, in terms of these lightlike vectors, as 
$\ell^{\mu}=(n \cdot \ell) \frac{\bar{n}^{\mu}}{2}+(\bar{n} \cdot \ell) \frac{n^{\mu}}{2}+\ell_{\perp}^{\mu}=\ell_{+} \frac{\bar{n}^{\mu}}{2}+\ell_{-} \frac{n^{\mu}}{2}+\ell_{\perp}^{\mu}$.

Here, $\ell_{\perp}=\left(0, \ell^{1}, \ell^{2}, \ldots, \ell^{D-2}, 0\right)$ is a $(D-2)$-dimensional vector, and we have defined

$$
\ell_{+} \equiv n \cdot \ell, \quad \ell_{-} \equiv \bar{n} \cdot \ell
$$

As a consequence, the scalar product of two vectors $k$ and $\ell$ becomes

$k \cdot \ell=\frac{k_{+} \ell_{-}}{2}+\frac{k_{-} \ell_{+}}{2}+k_{\perp} \cdot \ell_{\perp}=\frac{k_{+} \ell_{-}}{2}+\frac{k_{-} \ell_{+}}{2}-\boldsymbol{k}_{\perp} \cdot \boldsymbol{\ell}_{\perp}$,

where $\boldsymbol{\ell}_{\perp}$ and $\boldsymbol{k}_{\perp}$ are $(D-2)$-dimensional Euclidean vectors.

We make use of the following SCET notations. $q_{s}$ is the soft-quark Dirac spinor, $G_{s}=T^{a} G_{s}^{a}$, where $G_{s}^{a}$ is the softgluon field with color index $a$ and $T^{a}$ is an $S U(3)$ color matrix in the fundamental representation, $G_{n}=T^{a} G_{n}^{a}$, where $G_{n}^{a}$ is an $n$-hard-collinear gluon field with color index $a, A_{n}$ is an $n$-hard-collinear photon field, $\xi_{n}$ is an $n$ hard-collinear-quark Dirac spinor, which satisfies $\not h \xi_{n}=0$, $i D_{n}^{\perp \mu}=i \partial^{\perp \mu}+g_{s} G_{n}^{\perp \mu}+e_{q} A_{n}^{\perp \mu}$ is a transverse covariant derivative, $g_{s}=\sqrt{4 \pi \alpha_{s}}$ is the strong coupling, and $e_{q}$ is the electric charge of the collinear quark.

The scaling of a soft momentum is given by

$$
k_{s}^{\mu} \sim(\lambda, \lambda, \lambda) Q
$$

Here $Q$ is the large momentum scale and $\lambda \sim m_{b} / Q$. We follow the convention

$$
k=\left(k_{+}, k_{-}, \boldsymbol{k}_{\perp}\right)
$$

for the light-front coordinates. The scaling of an $n$-hardcollinear momentum is given by

$$
k_{n}^{\mu} \sim\left(\lambda, 1, \lambda^{\frac{1}{2}}\right) Q .
$$

The soft-quark spinor scales as $\lambda^{\frac{3}{2}}$, the soft-gluon field scales as $\lambda$, the $n$-hard-collinear spinors scale as $\lambda^{\frac{1}{2}}$, and the $n$-hard-collinear gauge fields scale as the $n$-hard-collinear momentum $k_{n}^{\mu}$ in Eq. (7) [17].

\section{RADIATIVE JET FUNCTION}

\section{A. Operator definition of the radiative jet function}

In the factorization theorem of Ref. [22], the interactions of virtual hard-collinear quarks and gauge bosons with an outgoing real hard-collinear photon are described by the radiative jet function. The radiative jet function couples to the soft quark by virtue of an interaction between the soft quark and the hard-collinear quarks and gauge fields that first appears at subleading order in the expansion of the SCET Lagrangian. This coupling is given, in the notation of [17], by

$$
\mathcal{L}_{q \xi_{n}}^{(1 / 2)}(x)=\bar{q}_{s}\left(x_{-}\right) W_{n}^{\dagger}(x) i \not D_{n}^{\perp} \xi_{n}(x)+\text { H.c. },
$$

where H.c. denotes the Hermitian-conjugate contributions. Here, the argument of the soft-quark field $q_{s}$ is taken to be $x_{-}$, with $x_{-}^{\mu}=(\bar{n} \cdot x) \frac{n^{\mu}}{2}$. This argument effects the expansion in momentum space at leading power in $\lambda$, in which the collinear subdiagrams depend only on the + component of the soft momentum. (This is the multipole expansion of SCET [17].) $W_{n}$ is a collinear Wilson line, which is defined by

$$
\begin{aligned}
W_{n}(x)= & P \exp \left[i g_{s} \int_{-\infty}^{0} d s \bar{n} \cdot G_{n}(x+s \bar{n})\right. \\
& \left.+i e_{q} \int_{-\infty}^{0} d s \bar{n} \cdot A_{n}(x+s \bar{n})\right]
\end{aligned}
$$

where $P$ denotes the path ordering of the exponential. We drop the term involving $A_{n}$ in the remainder of this paper.

In discussing the soft subtraction, we will encounter the soft Wilson line along $n$ which is defined by

$$
S_{n}(x)=P \exp \left[i g_{s} \int_{-\infty}^{0} d t n \cdot G_{s}(x+t n)\right] .
$$

We take the definition of the radiative jet function $J\left(p^{2}\right)$ that is given in Eq. (1.2) of Ref. [25]:

$$
\begin{aligned}
\bar{J}\left(\bar{n} \cdot p, p^{2}\right) & \equiv i \int d^{D} x e^{i \frac{i_{+} x_{-}}{2}} \\
& \times\left\langle\gamma\left(k_{1}\right)\left|T\left(W_{n}^{\dagger} i \not_{n}^{\perp} \xi_{n}\right)^{a}(x)\left(\bar{\xi}_{n} W_{n}\right)^{b}(0)\right| 0\right\rangle \\
& =i e_{q} \delta^{a b} \notin_{\perp}^{*}\left(k_{1}\right) \frac{\not h}{2} \frac{i \bar{n} \cdot p}{p^{2}+i \varepsilon} J\left(p^{2}\right) .
\end{aligned}
$$

Here, we have made the color indices $a$ and $b$ explicit, and we have defined $\bar{J}\left(\bar{n} \cdot p, p^{2}\right)$ to be the complete expression in Eq. (11). $\gamma\left(k_{1}\right)$ denotes a real photon with momentum $k_{1}=\left(0, k_{1-}, \mathbf{0}_{\perp}\right)$ and polarization $\varepsilon^{*}\left(k_{1}\right)$, and $T$ denotes the time-ordered product. We have inserted a factor $i$ in front of the matrix element in Eq. (11), so that there is a factor $i$ that is associated with each real-photon vertex, whether it arises from the covariant derivative in the matrix element or from the QED interaction Lagrangian. $p$ is defined by

$$
p^{\mu} \equiv k_{1}^{\mu}+\ell^{\mu},
$$

and $\ell$ has the interpretation of the soft momentum that is carried by the soft quark in Eq. (8). Note that

$$
\ell_{+}=p_{+}
$$


Throughout this paper, we take the approximation

$$
\ell^{\mu} \approx \ell_{+} \frac{\bar{n}^{\mu}}{2}
$$

Since $k_{1}$ satisfies hard-collinear scaling and $\ell$ satisfies soft scaling, this approximation gives the leading power in $\lambda$ in the argument $p^{2}$ of the radiative jet function. This is the multipole expansion to which we alluded earlier. It follows that

$$
p^{2} \approx k_{1-} \ell_{+}=p_{-} p_{+}
$$

This approximation has been invoked in writing $p^{2}$ in the arguments of $\bar{J}$ and $J$. In the remainder of this paper, we suppress the argument $\bar{n} \cdot p \approx k_{1-}$ in $\bar{J}$.

$\bar{J}\left(p^{2}\right)$ contains contributions in which the real photon attaches to the covariant derivative and contributions in which the real photon attaches to the quark line. Following Ref. [22], we call the former contributions $\bar{J}_{A}\left(p^{2}\right)$, and we call the latter contributions $\bar{J}_{G}\left(p^{2}\right)$, writing

$$
\bar{J}\left(p^{2}\right)=\bar{J}_{A}\left(p^{2}\right)+\bar{J}_{G}\left(p^{2}\right) .
$$

In computing contributions to the radiative jet function in this paper, we do not use the SCET Feynman rules. Instead, we follow a procedure that is equivalent, but more amenable to a graphical analysis. Starting from the Feynman rules for QCD, we insert projectors

$$
\begin{aligned}
& P_{n}=\frac{\not h \hbar}{4}, \\
& P_{\bar{n}}=\frac{\hbar m}{4}
\end{aligned}
$$

on the outgoing and incoming ends of the quark lines, respectively, so as to obtain the components of the Dirac spinor that are large in $n$-hard-collinear scaling and that correspond to the $n$-hard-collinear spinors $\xi_{n}$ and $\bar{\xi}_{n}$, respectively.

\section{B. Subtraction and factorization of soft contributions to the radiative jet function}

The radiative jet function, as defined in Eq. (11), also contains soft contributions. These must be removed in order to avoid double counting of contributions in the soft function in the factorization theorems. One can factor the soft contributions from the jet function by making use of standard techniques from the diagrammatic methods for proving factorization theorems [31]. We carry out the factorization in the Feynman gauge. However, the resulting expressions are gauge invariant. Our approach in dealing with the soft subtractions is analogous to the one that is given in Sec. 10.8.7 of Ref. [27].
Soft divergences can develop if one end of a soft gluon attaches either to a Wilson line or to a soft quark line and the other end attaches to an $n$-hard-collinear line. There is no soft divergence if both ends of a gluon attach to $n$-hardcollinear lines. If both ends of a soft gluon attach to a soft quark line, then that soft divergence is part of the soft function or is internal to a soft subtraction, and no special treatment of it is required.

Let us consider first the case of soft gluons that attach to hard-collinear lines, but not to the Wilson lines $W_{n}$ and $W_{n}^{\dagger}$. At leading order in the scaling parameter $\lambda$, the current $j^{\mu}$ in the hard-collinear lines to which the soft gluons couple is proportional $n^{\mu}$. It follows that, in the attachment of that soft gluon to a hard-collinear line, its polarization sum $g^{\mu \nu}$ can be replaced with $k^{\nu} n^{\mu} /(n \cdot k+i \varepsilon)$, where the $i \varepsilon$ prescription corresponds to a momentum routing in which $k$ flows parallel to the arrow on the quark propagator. Note that the polarization now corresponds to a pure gauge. This is the Grammer-Yennie approximation [29]. Then, one can apply graphical Ward identities to show that sum over the attachments of all such soft gluons to the hard-collinear lines can be replaced with the sum over all attachments of the soft gluons to Wilson lines $S_{n}$ and $S_{n}^{\dagger}$ that attach to the quark line immediately to the outgoing side and immediately to the incoming side, respectively, of the outermost hard-collineargluon attachments. Details of this step are given following Eq. (4.3) of Ref. [30]. In SCET, this step can be implemented by making use of field redefinitions [13].

In carrying out this analysis, we omit diagrams that contain $n$-hard-collinear subdiagrams that are not connected by lines carrying $n$-hard-collinear momenta to the external photon. These diagrams lead to contributions that are not properly part of the radiative jet function because the disconnected subdiagram does not yield a pinch singularity in the $n$-hard-collinear momentum region. (At one-loop order, these contributions vanish in dimensional regularization.) Such diagrams contain quark lines that carry soft momenta and, so, if they were to contribute to the radiative jet function, they would cause the GrammerYennie approximation and the factorization of the soft subtractions to fail. We will discuss an example of such a diagram in Sec. VI B.

Next, let us consider the case of soft gluons that attach to the collinear Wilson lines $W_{n}$ and $W_{n}^{\dagger}$. Here, we use the fact that soft-gluon attachments to the collinear Wilson lines $W_{n}$ and $W_{n}^{\dagger}$ that lie to the outside of all collinear-gluon attachments factor into new soft Wilson lines $S_{\bar{n}}$ and $S_{\bar{n}}^{\dagger}$, respectively, that lie to the outside of the $W_{n}$ and $W_{n}^{\dagger}$ collinear Wilson lines of the jet function [30]. ${ }^{4}$ Soft-gluon attachments to the collinear Wilson lines $W_{n}$ and $W_{n}^{\dagger}$ that

\footnotetext{
${ }^{4}$ Note that the collinear Wilson line $W_{n}$ contains projections of the gluon field onto $\bar{n}$. [See Eq. (9).] Hence, $W_{n}$ gives rise to $S_{\bar{n}}$, and $W_{n}^{\dagger}$ gives rise to $S_{\bar{n}}^{\dagger}$.
} 

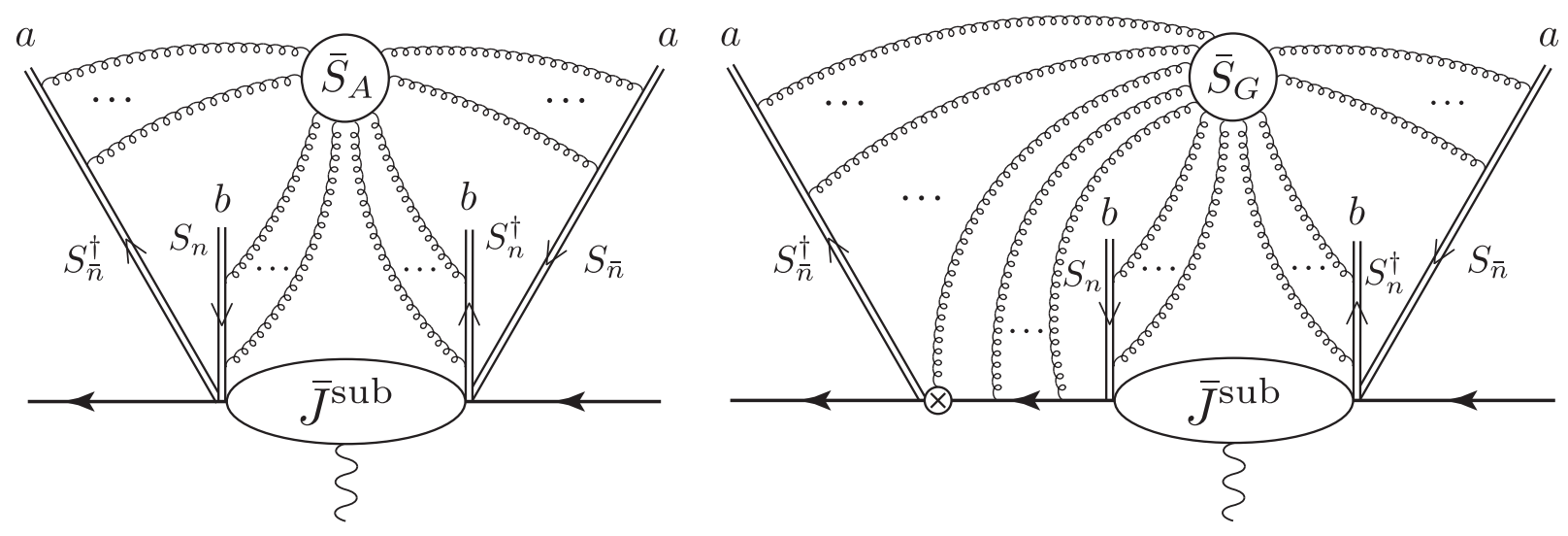

FIG. 2. Diagrammatic representation of the extraction of the soft contributions from $\bar{J}$. $a$ and $b$ are color indices.

lie to the inside of collinear-gluon attachments yield powersuppressed contributions [30].

The resulting diagrams have the form of those in Fig. 2. At this stage, the blobs labeled $\bar{J}^{\text {sub }}$ contain all of the collinear contributions and contain no soft contributions. The first diagram in Fig. 2, whose soft subtractions we denote by $\bar{S}_{A}$, arises from the contributions in which no soft gluons attach to the quark line to the outgoing side of the collinear-gluon attachments. The second diagram in Fig. 2, whose soft subtractions we denote by $\bar{S}_{G}$, arises from the contributions in which one or more soft gluons attach to the quark line to the outgoing side of the collinear-gluon attachments. ${ }^{5}$

We call the quark line that lies between the covariant derivative and $S_{n}$ in the second diagram in Fig. 2 the $n$-hard-collinear-soft quark line. Its Feynman rules are obtained from those for the $n$-hard-collinear quark line by retaining only those contributions that are leading in the soft scaling of the gluon momenta. An $n$-hard-collinear quark line couples to a soft quark line only through the covariant derivative or $W_{n}^{\dagger}$ in Eq. (8), and at least one $n$-hard-collinear gluon must attach to the covariant derivative or to $W_{n}^{\dagger}$ in order to produce an $n$-hard-collinear momentum in the quark line. Therefore, an $n$-hardcollinear-soft quark line couples to a soft quark line only through the covariant derivative or $S_{\bar{n}}^{\dagger}$, and at least one soft gluon must attach to the covariant derivative or to $S_{\bar{n}}^{\dagger}$.

The blob in the first diagram of Fig. 2 is in the form of a radiative jet function, except that it also contains soft subtractions, which can be implemented by subtracting the Grammer-Yennie form from specific gluon vertices. The blob in the second diagram of Fig. 2 requires some rearrangement to put it into the form of a radiative jet function (with soft subtractions). One approach is simply to

\footnotetext{
${ }^{5}$ Note that there is not a one-to-one correspondence between $\bar{S}_{A}$ and $\bar{J}_{A}$ or between $\bar{S}_{G}$ and $\bar{J}_{G} . \bar{J}_{A}$ decomposes into $\bar{S}_{A} \otimes \bar{J}_{A}^{\text {sub }}$, but $\bar{J}_{G}$ decomposes into $\bar{S}_{A} \otimes \bar{J}_{G}^{\text {sub }}, \bar{S}_{G} \otimes \bar{J}_{G}^{\text {sub }}$, and $\bar{S}_{G} \otimes \bar{J}_{A}^{\text {sub }}$.
}

invoke the form of the SCET Lagrangian for $n$-hardcollinear quarks and gluons and their couplings to a soft quark and a real photon to deduce that the blob in the second diagram of Fig. 2 takes the form of a radiative jet function (with soft subtractions) in SCET. Here, it is important that we have defined $\bar{J}$ in Eq. (11) in such a way that the vertex for a transverse photon is always $i e_{q} \gamma_{\perp}^{\mu}$, regardless of whether the photon attaches to a quark line or to a covariant derivative. In the Appendix, we sketch how the rearrangement of the blob in the second diagram of Fig. 2 into the radiative-jet form can be achieved in the diagrammatic approach.

We remind the reader that, owing to the multipole expansion, we keep only the plus components of soft momenta that enter $\bar{J}^{\text {sub }}$. Using this fact, we can express the diagrams of Fig. 2, as convolutions over the plus component of momentum:

$\bar{J}\left(p_{-} p_{+}\right)=\int \frac{d k_{+}}{p_{+}} \bar{S}\left(k_{+}\right) \bar{J}^{\mathrm{sub}}\left[p_{-}\left(p_{+}-k_{+}\right)\right] \equiv \bar{S} \otimes \bar{J}^{\mathrm{sub}}$

Note that we can also write this convolution in the form

$$
\bar{S} \otimes \bar{J}^{\mathrm{sub}}=\int d x \bar{S}\left[(1-x) p_{+}\right] \bar{J}^{\mathrm{sub}}\left(x p^{2}\right),
$$

where we have made the variable change

$$
k_{+}=(1-x) p_{+},
$$

and we have used $p^{2} \approx p_{+} p_{-}$[Eq. (15)]. Because both $\bar{S}$ and $\bar{J}^{\text {sub }}$ are Dirac matrices, the order of the factors in the convolution is significant.

The soft-subtraction function is given by

$$
\bar{S}\left(k_{+}\right)=\bar{S}_{A}\left(k_{+}\right)+\bar{S}_{G}\left(k_{+}\right)
$$

where 


$$
\begin{aligned}
& \bar{S}_{A}\left(k_{+}\right)=\frac{p_{+}}{2 \pi N_{c}} \int \frac{d x_{-}}{2} e^{i \frac{k_{+} x_{-}}{2}}\left\langle 0\left|T\left(S_{\bar{n}}^{\dagger} S_{n}\right)^{a b}\left(x_{-}\right)\left(S_{n}^{\dagger} S_{\bar{n}}\right)^{b a}(0)\right| 0\right\rangle, \\
& \bar{S}_{G}\left(k_{+}\right)=\frac{i p_{+}}{2 \pi N_{c}} \int d^{D} y \int \frac{d x_{-}}{2} e^{i \frac{p_{+} y_{-}}{2}} e^{i \frac{\left(k_{+}-p_{+}\right) x_{-}}{2}}\left\langle 0\left|T\left(S_{\bar{n}}^{\dagger} P_{n} i \not D_{s}^{\perp} \psi_{n, s}\right)^{a}(y)\left(\bar{\psi}_{n, s} P_{n} S_{n}\right)^{b}\left(x_{-}\right)\left(S_{n}^{\dagger} S_{\bar{n}}\right)^{b a}(0)\right| 0\right\rangle .
\end{aligned}
$$

In the arguments of $\bar{S}_{A}$ and $\bar{S}_{G}$, we have suppressed the dependences on the parameter $p_{+}=\ell_{+}$. The covariant derivative is labeled with a subscript $s$ as a reminder that the gauge fields and their momenta in the covariant derivative satisfy soft scaling. $\psi_{n, s}$ is the $n$-hard-collinear-soft field, which corresponds to the $n$-hard-collinear-soft line in the second diagram in Fig. 2 that we have described earlier. The subscript $n, s$ is a reminder that the propagator and interactions of this field with soft gauge fields are obtained by first taking an approximation in which the quark momentum and the momenta of the attached gauge fields have $n$-hard-collinear scaling and then taking an approximation in which the quark momentum and the momenta of the attached gauge fields have soft scaling. One consequence of this is that the propagator for $\psi_{n, s}$ is massless.

In the equation for $\bar{S}_{G}$, the left-hand factor $P_{n}$ arises from the factor $P_{n}$ in the unsubtracted radiative jet function $\bar{J}$, while the right-hand factor $P_{n}$ arises from applying the identity $P_{n}=P_{n}^{2}$ to the factor $P_{n}$ on the left side of $\bar{J}^{\text {sub }}$ in Eq. (18). As we have mentioned, at least one soft gluon must attach to the covariant derivative or to $S_{\bar{n}}^{\dagger}$ in $\bar{S}_{G}$. Consequently, one should subtract the noninteracting part, that is, make the replacement $S_{\bar{n}}^{\dagger} i \not D_{s}^{\perp} \rightarrow S_{\bar{n}}^{\dagger} i \not D_{s}^{\perp}-i \not D^{\perp}$.

At any order in perturbation theory, the soft-subtraction contributions can also be obtained by starting with the jet contributions and retaining only the parts that have leading soft scaling [Eq. (5)]. However, in this form, it is not apparent that the soft subtractions factor from the radiative jet function to yield the form in Eq. (21). As we will see, it is the form in Eq. (21) that leads to the nonlocality in the renormalization of the radiative jet function.

It follows from Eq. (18) that $\bar{J}^{\text {sub }}$ is given by

$$
\bar{J}^{\mathrm{sub}}=\bar{S}^{-1} \otimes \bar{J} .
$$

The quantity $\bar{S}^{-1}$ can be obtained to any order in $\alpha_{s}$ by making use of the expansion of $\bar{S}$ in powers of $\alpha_{s}$, namely,

$$
\bar{S}=1+\alpha_{S} \bar{S}^{(1)}+\ldots,
$$

and solving the equation

$$
\bar{S}^{-1} \otimes \bar{S}=1
$$

iteratively. Specifically, in order $\alpha_{s}$, Eq. (22) can be written in the form

$$
\bar{J}^{\mathrm{sub}}=\left(1-\alpha_{s} \bar{S}^{(1)}\right) \otimes \bar{J} .
$$

\section{RENORMALIZATION OF THE RADIATIVE JET FUNCTION}

The renormalization of $\bar{J}^{\text {sub }}$ arises from two sources; the radiative jet function and the soft function.

The radiative jet function $\bar{J}$ in Eq. (18) is multiplicatively renormalized,

$$
\bar{J}_{R}\left(p^{2} ; \mu\right)=Z_{\bar{J}}\left(p^{2} ; \mu\right) \bar{J}\left(p^{2}\right),
$$

where $\mu$ is the renormalization scale. That is, the renormalization of $\bar{J}$ is local. This follows from the fact, in order for there to be a nonlocal renormalization for $\bar{J}$, a UV-divergent diagrammatic loop must transfer a plus component of loop momentum $k_{+}$from one external leg of $\bar{J}$ to the other external leg of $\bar{J}$. However, a loop that transfers $k_{+}$in this way does not have a UV-divergent power count because there are too many propagators in the loop. We will see explicit examples of this in the one-loop calculations in Sec. VI.

The soft-subtraction function $\bar{S}$ is nonlocally renormalized:

$\bar{S}\left(k_{+}\right)=\int \frac{d k_{+}^{\prime}}{k_{+}} \bar{S}_{R}\left(k_{+}-k_{+}^{\prime} ; \mu\right) Z_{\bar{S}}^{-1}\left(k_{+}^{\prime} / k_{+} ; \mu\right) \equiv \bar{S}_{R} \otimes Z_{\bar{S}}^{-1}$.

In contrast with $\bar{J}, \bar{S}$ can have nonlocal renormalizations because two of the operators in the definitions of $\bar{S}$ in Eq. (21) are separated only in the minus direction. That is, in momentum space, only the plus component of a loop momentum that routes through these operators is constrained. Consequently, a loop momentum $k$ can transfer $k_{+}$from one external leg of $\bar{S}$ to the other external leg, and the integration over $\boldsymbol{k}_{\perp}$ can still be UV divergent. We will also see explicit examples of this phenomenon in the one-loop calculation in Sec. VI.

Note that we can also write the convolution in the form

$$
\bar{S}\left(k_{+}\right)=\int d x^{\prime} \bar{S}_{R}\left(x^{\prime} k_{+} ; \mu\right) Z_{\bar{S}}^{-1}\left(1-x^{\prime} ; \mu\right),
$$

where we have used $k_{+}^{\prime}=\left(1-x^{\prime}\right) k_{+}$. Under the change of integration variables $x^{\prime}=1-x$, the convolution in Eq. (27) is identical to the one in Eq. (18), aside from a trivial rescaling of the argument of $Z_{\bar{S}}^{-1}$ with a factor of $p^{2}$ and the 
argument of $\bar{S}_{R}$ with a factor $p_{+} / k_{+}$. Therefore, we use the same notation $(\otimes)$ for both convolutions. However, one should keep in mind the rescalings of arguments that are implicit in this notation. It follows that

$$
\begin{aligned}
\bar{J}^{\text {sub }} & =\bar{S}^{-1} \otimes \bar{J}=\left(\bar{S}_{R} \otimes Z_{\bar{S}}^{-1}\right)^{-1} \otimes Z_{\bar{J}}^{-1} \bar{J}_{R} \\
& =Z_{\bar{J}}^{-1} Z_{\bar{S}} \otimes \bar{S}_{R}^{-1} \otimes \bar{J}_{R} \\
& =Z_{\bar{J}}^{-1} Z_{\bar{S}} \otimes \bar{J}_{R}^{\text {sub }}
\end{aligned}
$$

This implies that

$$
\begin{aligned}
& \bar{J}_{R}^{\mathrm{sub}}\left(p_{-} p_{+} ; \mu\right) \\
& =\left(Z_{\bar{J}^{\text {sub }}} \otimes \bar{J}^{\text {sub }}\right)\left(p_{-} p_{+} ; \mu\right) \\
& =\int \frac{d k_{+}}{p_{+}} Z_{\bar{J}^{\text {sub }}}\left(k_{+} / p_{+}, p^{2} ; \mu\right) \bar{J}^{\mathrm{sub}}\left[p_{-}\left(p_{+}-k_{+}\right)\right],
\end{aligned}
$$

or, equivalently,

$\bar{J}_{R}^{\mathrm{sub}}\left(p^{2} ; \mu\right)=\int d x Z_{\bar{J}^{\text {sub }}}\left[(1-x), p^{2} ; \mu\right] \bar{J}^{\mathrm{sub}}\left(x p^{2}\right)$,

where

$$
Z_{\bar{J} \text { sub }}\left[(1-x), p^{2} ; \mu\right]=Z_{\bar{J}}\left(p^{2} ; \mu\right) Z_{\bar{S}}^{-1}[(1-x) ; \mu],
$$

and we have used $p^{2} \approx p_{+} p_{-}$. Note that, the renormalization factors $Z_{\bar{J}}$ and $Z_{\bar{S}}^{-1}$ contain only those renormalizations that are associated with the operator matrix element (operator renormalizations) and do not contain the mass and coupling-constant renormalizations of QCD, except for the quark wave-function renormalization.

In order to make contact with the renormalization of the radiative jet function in Ref. [25], we make use of Eq. (31) and the relation between $J\left(p^{2}\right)$ and $\bar{J}\left(p^{2}\right)$ in Eq. (11) to obtain

$$
J_{R}^{\mathrm{sub}}=Z_{J^{\mathrm{sub}}} \otimes J^{\mathrm{sub}},
$$

where

$$
Z_{J^{\text {sub }}}\left[(1-x), p^{2} ; \mu\right]=(1 / x) Z_{\bar{J}^{\mathrm{sub}}}\left[(1-x), p^{2} ; \mu\right] .
$$

\section{LOWEST-ORDER EXPRESSIONS}

From the definition of $\bar{J}$ in Eq. (11), it follows that the lowest-order expression for $\bar{J}$ is given by

$$
\bar{J}^{(0)}\left(p^{2}\right)=i e_{q} \delta^{a b} \phi_{\perp}^{*}\left(k_{1}\right) \frac{\not h}{2} \frac{i \bar{n} \cdot p}{p^{2}+i \varepsilon} J^{(0)}\left(p^{2}\right),
$$

where $J^{(0)}\left(p^{2}\right)=1$ is the contribution to $J\left(p^{2}\right)$ at leading order in $\alpha_{s}$. From the definition of the soft-subtraction function in Eq. (21), we find that the lowest-order expressions for $\bar{S}_{A}$ and $\bar{S}_{G}$ are given by

$$
\begin{aligned}
& \bar{S}_{A}^{(0)}\left(k_{+}\right)=p_{+} \delta\left(k_{+}\right), \\
& \bar{S}_{G}^{(0)}\left(k_{+}\right)=0 .
\end{aligned}
$$

Then, making use of the relation in Eq. (18), we find that the lowest-order expression for $\bar{J}^{\text {sub }}$ is the same as $\bar{J}^{(0)}$ :

$$
\begin{aligned}
\bar{J}^{(0)}\left(p^{2}\right) & =\int \frac{d k_{+}}{p_{+}} \bar{S}_{A}^{(0)}\left(k_{+}\right) \bar{J}^{\operatorname{sub}(0)}\left[p_{-}\left(p_{+}-k_{+}\right)\right] \\
& =\bar{J}^{\operatorname{sub}(0)}\left(p^{2}\right) .
\end{aligned}
$$

Note also that

$$
\begin{aligned}
{\left[\bar{S}_{R}^{(0)}\right.} & \left.\otimes\left(Z_{\bar{S}}^{-1}\right)^{(1)}\right] \otimes \bar{J}^{\text {sub }} \\
& =\left(Z_{\bar{S}}^{-1}\right)^{(1)} \otimes \bar{J}^{\text {sub }} \\
& =\int \frac{d k_{+}}{p_{+}}\left(Z_{\bar{S}}^{-1}\right)^{(1)}\left(k_{+} / p_{+}\right) \bar{J}^{\mathrm{sub}}\left[p_{-}\left(p_{+}-k_{+}\right)\right] \\
& =\int d x\left(Z_{\bar{S}}^{-1}\right)^{(1)}(1-x) \bar{J}^{\mathrm{sub}}\left(x p^{2}\right),
\end{aligned}
$$

where we have used $\bar{S}_{R}^{(0)}=\bar{S}_{A}^{(0)}$ in the first equality and used $k_{+}=(1-x) p_{+}$and $p^{2} \approx p_{+} p_{-}$in the last equality. We make use of Eq. (36) in computing the order- $\alpha_{s}$ contribution to $Z_{\bar{S}}^{-1}$ in Sec. VI.

Finally, we have for the lowest-order contribution to $Z_{\bar{S}}^{-1}$

$$
\left(Z_{\bar{S}}^{-1}\right)^{(0)}(1-x)=\delta(1-x) .
$$

\section{RENORMALIZATION OF $\bar{J}^{\text {sub }}\left(p^{2}\right)$ AT ORDER $\alpha_{s}$}

In this section we compute the renormalization of the subtracted radiative jet function in order $\alpha_{S}$.

$Z_{\bar{S}}$ and $Z_{\bar{J}}$ have expansions in powers of $\alpha_{s}$ :

$$
\begin{aligned}
& Z_{\bar{S}}=\mathbf{1}+\alpha_{s} Z_{\bar{S}}^{(1)}+\ldots, \\
& Z_{\bar{J}}=1+\alpha_{s} Z_{\bar{J}}^{(1)}+\ldots
\end{aligned}
$$

Here, 1 represents the lowest-order contribution to $Z_{\bar{S}}$ [Eq. (37)]. Then, from Eq. (31b) we find that, to order $\alpha_{s}$ in the renormalizations, $\bar{J}_{R}^{\text {sub }}$ is given by

$$
\bar{J}_{R}^{\text {sub }}=\left[\mathbf{1}+\alpha_{s} Z_{\bar{J}^{\text {sub }}}^{(1)}+O\left(\alpha_{s}^{2}\right)\right] \otimes \bar{J}^{\text {sub }}
$$

where 
$Z_{\bar{J}^{\text {sub }}}^{(1)}\left(1-x, p^{2} ; \mu\right)=Z_{\bar{J}}^{(1)}\left(p^{2} ; \mu\right) \delta(1-x)-Z_{\bar{S}}^{(1)}[(1-x) ; \mu]$.

Equation (39) is the basis for our order- $\alpha_{s}$ calculations of the renormalization of $\bar{J}^{\text {sub }}$.

The $Z$ factors in Eq. (39) are ultimately convolved with $\bar{J}^{\text {sub }}$. [See Eq. (36).] In extracting the UV divergences, we implement these convolutions (a simple multiplication in the case of $Z_{\bar{J}}$ ) in order to keep track of the nonlocality of the $Z_{\bar{S}}$ and in order to make use of the Dirac projector in $\bar{J}^{\text {sub }}$ to simplify expressions.

We compute the renormalization factor $Z_{\bar{J}}^{(1)}$ that is associated with the unsubtracted radiative jet function $\bar{J}$ [Eq. (11)] by evaluating the order- $\alpha_{s}$ UV divergence in $\bar{J}$, namely $\left(Z_{\bar{J}}^{-1}\right)^{(1)}$ and using $\left(Z_{\bar{J}}^{-1}\right)^{(1)}=-Z_{\bar{J}}^{(1)}$. We convolve the result with $\bar{J}^{\text {sub }}$.

We compute the renormalization factor $Z_{\bar{S}}^{(1)}$ that is associated with the soft subtraction [Eq. (21)] by evaluating $\left[\bar{S}_{R}^{(0)} \otimes\left(Z_{\bar{S}}^{-1}\right)^{(1)}\right] \otimes \bar{J}^{\text {sub }}$. Then, we make use of Eq. (36) to eliminate the trivial convolution involving $\bar{S}_{R}^{(0)}$, and we use
$\left(Z_{\bar{S}}^{-1}\right)^{(1)}=-Z_{\bar{S}}^{(1)}$. Finally, we combine this result with the result for $\left(Z_{\bar{J}}^{-1}\right)^{(1)} \otimes \bar{J}^{\text {sub }}$ according to Eq. (39).

We evaluate many of the integrals by using contour integration. In these evaluations, we take, for definiteness, $p_{+}>0$. This choice corresponds to a timelike argument $p^{2}>0$ in the radiative jet function because $p^{2} \approx p_{+} p_{-}$and $p_{-} \approx k_{1-}$ is always positive. However, our result for the anomalous dimension of the radiative jet function in the timelike case can be continued analytically to obtain the anomalous dimension in the spacelike $\left(p^{2}<0\right)$ case.

We carry out the computation in the Feynman gauge.

Feynman diagrams that potentially contribute in order $\alpha_{s}$ to the renormalization of the radiative jet function $\bar{J}$ in the Feynman gauge are shown in Fig. 3. Feynman diagrams that potentially contribute in order $\alpha_{s}$ to the renormalization of the soft subtractions in the Feynman gauge are shown in Fig. 4. In Fig. 4, the blobs represent $\bar{J}^{\text {sub }}$, which, as we have mentioned, we make explicit in order to keep track of the nonlocal nature of $Z_{\bar{S}}$ and the Dirac algebra.

In carrying out these computations we make use of the Feynman rules for the Wilson lines, which can be obtained from the definitions in Eqs. (9) and (10). A $W_{n}$ vertex contributes a factor $i g_{s} T^{a} \bar{n}^{\mu}$. A $W_{n}$ propagator contributes a factor

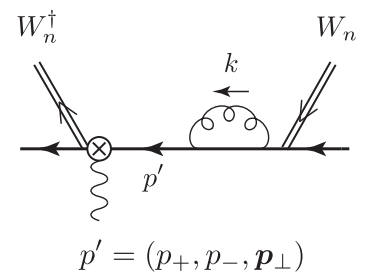

(a)

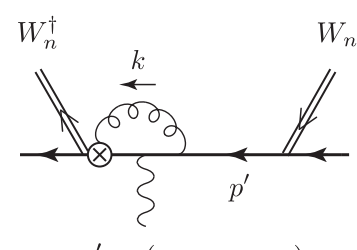

$p^{\prime}=\left(p_{+}, p_{-}, \boldsymbol{p}_{\perp}\right)$

(e)

$p^{\prime}=\left(p_{+}-k_{+}, p_{-}-k_{-}, \boldsymbol{p}_{\perp}-\boldsymbol{k}_{\perp}\right)$

(i)

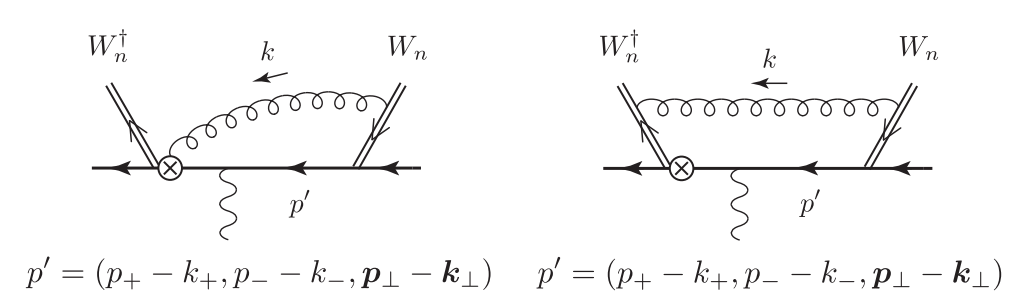

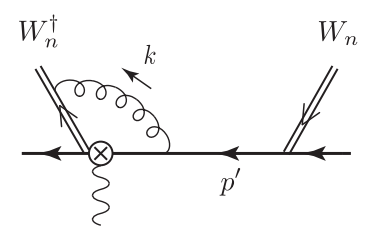

$p^{\prime}=\left(p_{+}, p_{-}, \boldsymbol{p}_{\perp}\right)$

(b)

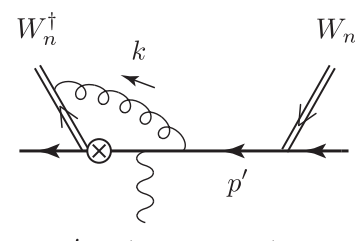

(f)

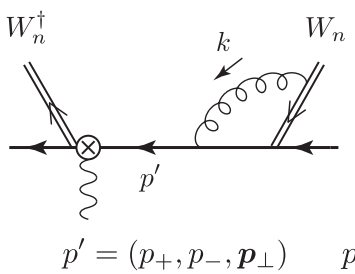

(c)

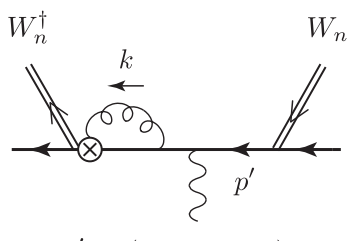

(g)

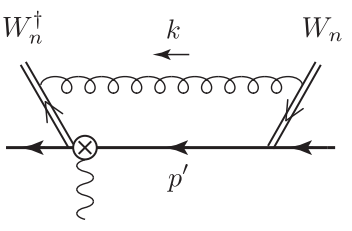

$p^{\prime}=\left(p_{+}-k_{+}, p_{-}-k_{-}, \boldsymbol{p}_{\perp}-\boldsymbol{k}_{\perp}\right)$

(d)

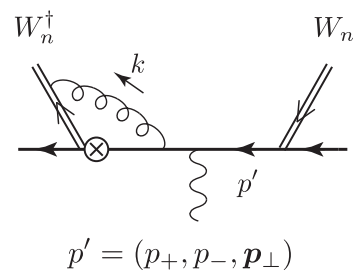

(h)

FIG. 3. Feynman diagrams that contribute order- $\alpha_{s}$ corrections to the radiative jet function in the Feynman gauge. The external momentum $p^{\prime}$ of the heavy-quark propagator is shown below each diagram. The diagrams in the first row arise from $\bar{J}_{A}$, and the diagrams in the second and third rows arise from $\bar{J}_{G}$. The crossed circle corresponds to the covariant derivative in Eq. (21). Our graphical notation differs from that in Ref. [22], in which the crossed circle represents both the covariant derivative and Wilson lines. 


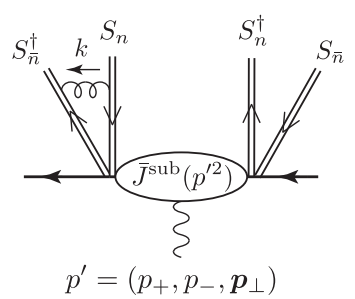

(a)

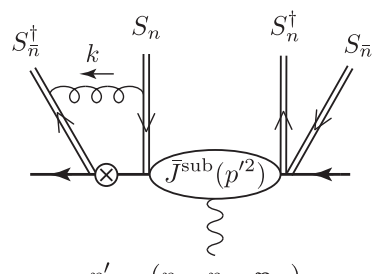

$p^{\prime}=\left(p_{+}, p_{-}, \boldsymbol{p}_{\perp}\right)$

(e)

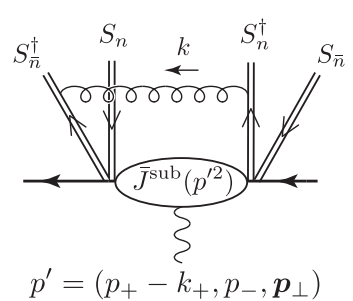

(b)

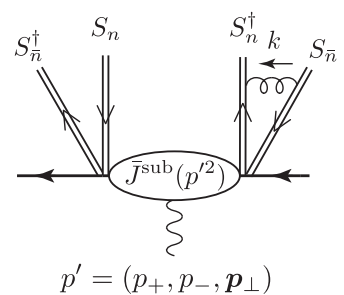

(c)

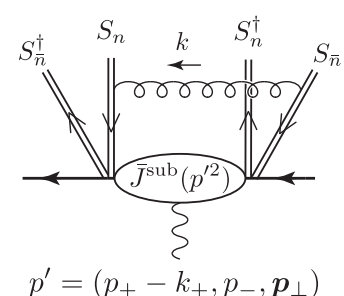

(d)

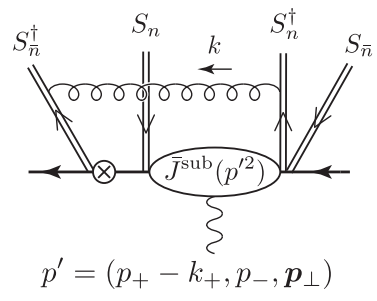

(f)

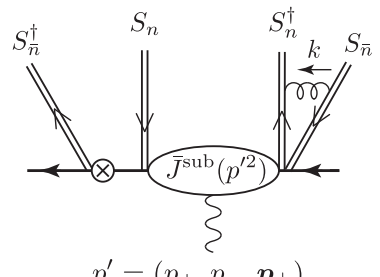

$p^{\prime}=\left(p_{+}, p_{-}, \boldsymbol{p}_{\perp}\right)$

(g)

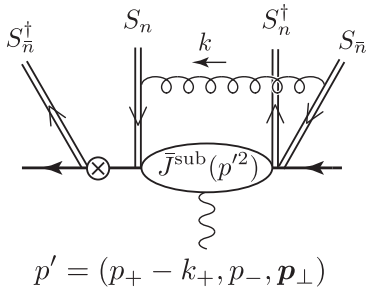

(h)

FIG. 4. Feynman diagrams that contribute to the renormalization of the soft-subtraction function $\bar{S}$ in order $\alpha_{s}$ in the Feynman gauge. The blobs in the diagrams represent $\bar{J}^{\mathrm{sub}}\left(p^{\prime 2}\right)$, and the corresponding $p^{\prime}$ is shown below each diagram. The diagrams (g) and (h) do not contribute, according to the definition of the soft-subtraction function that is given in Sec. III, because no soft gluons attach either to the covariant derivative or to $S_{\bar{n}}^{\dagger}$.

$$
\frac{i}{ \pm \bar{n} \cdot k+i \epsilon}
$$

The plus sign applies when $k$ flows into the Wilson line, and the minus sign applies when $k$ flows out of the Wilson line. In the case of $W_{\bar{n}}, \bar{n}$ is replaced with $n$. The Feynman rules for $W_{n}^{\dagger}$ and $W_{\bar{n}}^{\dagger}$ can be obtained from those for $W_{n}$ and $W_{\bar{n}}$, respectively, by taking the Hermitian conjugate and reversing the sign of the momentum in the propagator. The Feynman rules for $S_{n}\left(S_{\bar{n}}\right)$ are identical to the Feynman rules for $W_{\bar{n}}\left(W_{n}\right)$.

\section{A. UV divergences of $\bar{J}_{A}^{\text {sub }}\left(p^{2}\right)$ at order $\alpha_{s}$}

Let us discuss the contributions to $\bar{J}_{A}^{\text {sub }}\left(p^{2}\right)$ first. The diagrams that contribute to $\bar{J}$ in order $\alpha_{s}$ are shown in
Fig. 3. Among the diagrams in Fig. 3, diagrams (a)-(d) are contributions to $\bar{J}_{A}\left(p^{2}\right)$.

Diagram (d), which could potentially yield a nonlocal renormalization, has a UV-convergent power count. This is an example of the general argument that we have given in Sec. IV that $\bar{J}$ has only local renormalizations. [Diagram (d) also gives a vanishing contribution, since the collinear gluon is connected to two collinear Wilson lines that are in the same direction, which leads to both a vanishing numerator and a vanishing $k_{+}$contour integral.]

Diagram (a) in Fig. 3 is simply the quark self-energy diagram, and the corresponding amplitude is given by

$$
\begin{aligned}
i \mathcal{A}_{(\mathrm{a})} & =i e_{q} g_{s}^{2} C_{F}\left(\frac{\mu^{2} e^{\gamma_{E}}}{4 \pi}\right)^{\epsilon} \int \frac{d^{D} k}{(2 \pi)^{D}} \frac{\phi_{\perp}^{*} P_{n} \not \gamma^{\mu}(\not p-\not k) \gamma_{\mu} \not p P_{\bar{n}}}{\left(p^{2}+i \varepsilon\right)^{2}\left(k^{2}+i \varepsilon\right)\left[(p-k)^{2}+i \varepsilon\right]} \\
& =-e_{q} g_{s}^{2} C_{F}\left(\frac{\mu^{2} e^{\gamma_{E}}}{4 \pi}\right)^{\epsilon} \phi_{\perp}{ }^{*} \frac{\not h}{2} \frac{i \bar{n} \cdot p}{p^{2}+i \varepsilon} \int \frac{d^{D} k}{(2 \pi)^{D}} \frac{(D-2)\left(1-\frac{n \cdot k}{\bar{n} \cdot p}\right)}{\left(k^{2}+i \varepsilon\right)\left[(p-k)^{2}+i \varepsilon\right]},
\end{aligned}
$$

where $C_{F}=\left(N_{c}^{2}-1\right) /\left(2 N_{c}\right)$ and $N_{c}$ is the number of colors.

There is no soft subtraction in Fig. 4 that corresponds to this contribution. This follows from the fact that, in Eq. (41), $(p-k)^{2}+i \varepsilon \simeq p^{2}-p_{-} k_{+}+i \varepsilon$ when $k$ is soft, which implies that there is no pinch that prevents the $k_{-}$ integration contour from being deformed out of the soft region. From the Dirac structure in Eq. (41), we can see that the associated UV divergence corresponds to a quark wavefunction renormalization. Extracting the UV divergence and convolving (multiplying) with $\bar{J}^{\text {sub }}$, we obtain

$$
\left(-Z_{\bar{J}}^{(1)} \otimes \bar{J}^{\mathrm{sub}}\right)_{(\mathrm{a})}=-\frac{\alpha_{s} C_{F}}{4 \pi} \frac{1}{\epsilon_{\mathrm{UV}}} \bar{J}^{\mathrm{sub}}\left(p^{2}\right) .
$$


The subscript UV indicates that the origin of the divergence is UV.

The amplitudes of diagrams (b) and (c) in Fig. 3 are given by

$$
\begin{aligned}
i \mathcal{A}_{(\mathrm{b})} & =-i e_{q} g_{s}^{2} C_{F}\left(\frac{\mu^{2} e^{\gamma_{E}}}{4 \pi}\right)^{\epsilon} \int \frac{d^{D} k}{(2 \pi)^{D}} \frac{\phi_{\perp}^{*} P_{n}(\not p-\not k) \hbar \not p P_{\bar{n}}}{\left(p^{2}+i \varepsilon\right)\left(k^{2}+i \varepsilon\right)\left[(p-k)^{2}+i \varepsilon\right](\bar{n} \cdot k+i \varepsilon)} \\
& =-2 e_{q} g_{s}^{2} C_{F}\left(\frac{\mu^{2} e^{\gamma_{E}}}{4 \pi}\right)^{\epsilon} \phi_{\perp}^{*} \frac{\not h}{2} \frac{i \bar{n} \cdot p}{p^{2}+i \varepsilon} \int \frac{d^{D} k}{(2 \pi)^{D}} \frac{\bar{n} \cdot(p-k)}{\left(k^{2}+i \varepsilon\right)\left[(p-k)^{2}+i \varepsilon\right](\bar{n} \cdot k+i \varepsilon)}, \\
i \mathcal{A}_{(\mathrm{c})} & =i e_{q} g_{s}^{2} C_{F}\left(\frac{\mu^{2} e^{\gamma_{E}}}{4 \pi}\right)^{\epsilon} \int \frac{d^{D} k}{(2 \pi)^{D}} \frac{\phi_{\perp}^{*} P_{n} \not p \hbar(\not p-\not k) P_{\bar{n}}}{\left(p^{2}+i \varepsilon\right)\left(k^{2}+i \varepsilon\right)\left[(p-k)^{2}+i \varepsilon\right](-\bar{n} \cdot k+i \varepsilon)} \\
& =2 e_{q} g_{s}^{2} C_{F}\left(\frac{\mu^{2} e^{\gamma_{E}}}{4 \pi}\right)^{\epsilon} \phi_{\perp}^{*} \frac{\not h}{2} \frac{i \bar{n} \cdot p}{p^{2}+i \varepsilon} \int \frac{d^{D} k}{(2 \pi)^{D}} \frac{\bar{n} \cdot(p-k)}{\left(k^{2}+i \varepsilon\right)\left[(p-k)^{2}+i \varepsilon\right](-\bar{n} \cdot k+i \varepsilon)} .
\end{aligned}
$$

We convolve (multiply) the UV-divergent contribution in Eq. (43) with $\bar{J}^{\text {sub }}$ to obtain

$$
\begin{aligned}
& \alpha_{s}\left(-Z_{\bar{J}}^{(1)} \otimes \bar{J}^{\mathrm{sub}}\right)_{(\mathrm{b})}=-2 i g_{s}^{2} C_{F}\left(\frac{\mu^{2} e^{\gamma_{E}}}{4 \pi}\right)^{\epsilon}\left[\int \frac{d^{D} k}{(2 \pi)^{D}} \frac{\bar{n} \cdot(k-p) \bar{J}^{\mathrm{sub}}\left(p^{2}\right)}{\left(k^{2}+i \varepsilon\right)\left[(p-k)^{2}+i \varepsilon\right](\bar{n} \cdot k+i \varepsilon)}\right]_{\mathrm{UV}}, \\
& \alpha_{s}\left(-Z_{\bar{J}}^{(1)} \otimes \bar{J}^{\mathrm{sub}}\right)_{(\mathrm{c})}=2 i g_{s}^{2} C_{F}\left(\frac{\mu^{2} e^{\gamma_{E}}}{4 \pi}\right)^{\epsilon}\left[\int \frac{d^{D} k}{(2 \pi)^{D}} \frac{\bar{n} \cdot(k-p) \bar{J}^{\mathrm{sub}}\left(p^{2}\right)}{\left(k^{2}+i \varepsilon\right)\left[(p-k)^{2}+i \varepsilon\right](-\bar{n} \cdot k+i \varepsilon)}\right]_{\mathrm{UV}} .
\end{aligned}
$$

Here, the subscript UV indicates that only the UV-divergent part of the expression is to be kept.

As we will explain, the soft subtractions that correspond to diagram (b) in Fig. 3 are given by diagrams (a) and (b) in Fig. 4, and the soft subtractions that correspond to diagram (c) in Fig. 3 are given by diagrams (c) and (d) in Fig. 4. After working out the numerator algebra, we obtain the following contributions:

$$
\begin{aligned}
& \alpha_{s}\left(-Z_{\bar{S}}^{(1)} \otimes \bar{J}^{\mathrm{sub}}\right)_{(\mathrm{a}+\mathrm{b})}=-i n \cdot \bar{n} g_{s}^{2} C_{F}\left(\frac{\mu^{2} e^{\gamma_{E}}}{4 \pi}\right)^{\epsilon}\left[\int \frac{d^{D} k}{(2 \pi)^{D}} \frac{\bar{J}^{\mathrm{sub}}\left(p^{2}\right)-\bar{J}^{\mathrm{sub}}\left(p^{2}-\frac{2(\bar{n} \cdot p)(n \cdot k)}{n \cdot \bar{n}}\right)}{\left(k^{2}+i \varepsilon\right)(n \cdot k-i \varepsilon)(\bar{n} \cdot k+i \varepsilon)}\right]_{\mathrm{UV}} \\
& \alpha_{S}\left(-Z_{\bar{S}}^{(1)} \otimes \bar{J}^{\mathrm{sub}}\right)_{(\mathrm{c}+\mathrm{d})}=-i n \cdot \bar{n} g_{s}^{2} C_{F}\left(\frac{\mu^{2} e^{\gamma_{E}}}{4 \pi}\right)^{\epsilon}\left[\int \frac{d^{D} k}{(2 \pi)^{D}} \frac{\bar{J}^{\mathrm{sub}}\left(p^{2}\right)-\bar{J}^{\mathrm{sub}}\left(p^{2}-\frac{2(\bar{n} \cdot p)(n \cdot k)}{n \cdot \bar{n}}\right)}{\left(k^{2}+i \varepsilon\right)(n \cdot k+i \varepsilon)(\bar{n} \cdot k-i \varepsilon)}\right]_{\mathrm{UV}}
\end{aligned}
$$

We can see, in the case of the lowest-order expression for $\bar{J}^{\text {sub }}$ in Eq. (35), that the expression on the right-hand side of Eq. (45) is equal to the soft-approximation of the expression on the right-hand side of Eq. (44). This is the sense in which these soft subtractions correspond to diagram (b) in Fig. 3. Hence, in the case of the lowestorder expression for $\bar{J}^{\text {sub }}$, the IR divergences in Eq. (45) cancel those in Eq. (44). However, this cancellation does not hold in general because Eq. (44) is missing some of the jet-function contributions whose IR divergences would be canceled by the soft-subtraction contribution in Eq. (45). Recall that the expression in Eq. (45) contains the contributions of many soft-subtraction subgraphs that are summed through the use of the graphical Ward identities, while the expression in Eq. (44) contains only the contributions from those subgraphs that yield the UV divergences that are associated with the radiative-jet operator matrix element.
We can see as well, in the case of the lowest-order expression for $\bar{J}^{\mathrm{sub}}$, that the nonlocal contributions on the right side of Eq. (45) vanish. However, this feature is also special to the lowest-order case.

Note that, if one carries out the integration over $k_{-}$in Eq. (45) by contour integration, the resulting expression contains a scaleless integral in $k_{\perp}$ that yields a factor $1 / \epsilon_{\mathrm{UV}}-1 / \epsilon_{\mathrm{IR}}$. Therefore, if one does not distinguish between UV and IR poles in dimensional regularization, the soft subtraction vanishes. Hence, its role is to convert nonlocal IR poles to nonlocal UV poles.

Owing to the presence of the Wilson-line denominators $k_{-} \pm i \varepsilon$, the expressions in Eqs. (44) and (45) develop rapidity divergences as $k_{+} / k_{-} \rightarrow \infty$. These rapidity divergences cancel in the difference between Eqs. (44) and (45). Therefore, in order to avoid introducing a rapidity regulator, we compute the expressions in Eqs. (44) and (45) together. Reparameterization invariance [32] (separate rescaling of $n$ and $\bar{n}$ ) guarantees that, aside from the overall 
kinematic factor $\bar{n} \cdot p$ [see Eq. (11)], the expressions in Eqs. (44) and (45) are functions only of $p^{2}$ or $\frac{(n \cdot p)(\bar{n} \cdot p)}{n \cdot \bar{n}} \approx p^{2}$. (We will see this explicitly below.) Therefore, once we have extracted the $k$-independent factor $\bar{n} \cdot p$, the remainder of the integrand is invariant under $p \rightarrow-p$, and we can make the variable change $k \rightarrow-k$ to find that

$$
\left(-Z_{\bar{J}}^{(1)} \otimes \bar{J}^{\mathrm{sub}}\right)_{(\mathrm{b})}-\left(-Z_{\bar{S}}^{(1)} \otimes \bar{J}^{\mathrm{sub}}\right)_{(\mathrm{a}+\mathrm{b})}=\left(-Z_{\bar{J}}^{(1)} \otimes \bar{J}^{\mathrm{sub}}\right)_{(\mathrm{c})}-\left(-Z_{\bar{S}}^{(1)} \otimes \bar{J}^{\mathrm{sub}}\right)_{(\mathrm{c}+\mathrm{d})} .
$$

Therefore, in the analysis of these contributions, we compute only the combination $\left(-Z_{\bar{J}}^{(1)} \otimes \bar{J}^{\text {sub }}\right)_{(\mathrm{b})}-$ $\left(-Z_{\bar{S}}^{(1)} \otimes \bar{J}^{\mathrm{sub}}\right)_{(\mathrm{a}+\mathrm{b})}$.

We extract the contribution

$$
I_{\mathrm{b}}\left(p^{2}\right)=-2 i g_{s}^{2} C_{F}\left(\frac{\mu^{2} e^{\gamma_{E}}}{4 \pi}\right)^{\epsilon} \int \frac{d^{D} k}{(2 \pi)^{D}} \frac{\bar{J}^{\mathrm{sub}}\left(p^{2}\right)}{\left(k^{2}+i \varepsilon\right)\left[(p-k)^{2}+i \varepsilon\right]}
$$

from $\left(-Z_{\bar{J}}^{(1)} \otimes \bar{J}^{\text {sub }}\right)_{(\mathrm{b})}$, which gives the UV pole

$$
I_{\mathrm{b}}^{\mathrm{UV}}\left(p^{2}\right)=\frac{\alpha_{s} C_{F}}{2 \pi} \frac{1}{\epsilon_{\mathrm{UV}}} \bar{J}^{\mathrm{sub}}\left(p^{2}\right)
$$

Then, we combine the remaining part of the integrals in $\left(-Z_{\bar{J}}^{(1)} \otimes \bar{J}^{\text {sub }}\right)_{(\mathrm{b})}$ and the soft subtractions $\left(-Z_{\bar{S}}^{(1)} \otimes \bar{J}^{\text {sub }}\right)_{(\mathrm{a}+\mathrm{b})}$ to obtain

$$
\begin{aligned}
I_{\mathrm{sub}}\left(p^{2}\right)= & -2 i g_{s}^{2} C_{F}\left(\frac{\mu^{2} e^{\gamma_{E}}}{4 \pi}\right)^{\epsilon} \int \frac{d^{D} k}{(2 \pi)^{D}}\left\{\frac{-p_{-} \bar{J}^{\mathrm{sub}}\left(p^{2}\right)}{\left(k^{2}+i \varepsilon\right)\left[(p-k)^{2}+i \varepsilon\right]\left(k_{-}+i \varepsilon\right)}-\frac{\bar{J}^{\mathrm{sub}}\left(p^{2}\right)-\bar{J}^{\mathrm{sub}}\left(p^{2}-p_{-} k_{+}\right)}{\left(k^{2}+i \varepsilon\right)\left(k_{+}-i \varepsilon\right)\left(k_{-}+i \varepsilon\right)}\right\} \\
= & -2 i g_{s}^{2} C_{F}\left(\frac{\mu^{2} e^{\gamma_{E}}}{4 \pi}\right)^{\epsilon} \int \frac{d^{D} k}{(2 \pi)^{D}}\left\{\frac{-p_{-} \bar{J}^{\mathrm{sub}}\left(p^{2}\right)}{\left(k^{2}+i \varepsilon\right)\left(k_{-}+i \varepsilon\right)}\left[\frac{1}{(p-k)^{2}+i \varepsilon}-\frac{1}{p^{2}-p_{-} k_{+}+i \varepsilon}\right]\right. \\
& \left.+\frac{1}{\left(k^{2}+i \varepsilon\right)\left(k_{+}-i \varepsilon\right)\left(k_{-}+i \varepsilon\right)}\left[\bar{J}^{\mathrm{sub}}\left(p^{2}-p_{-} k_{+}\right)-\frac{p^{2} \bar{J}^{\mathrm{sub}}\left(p^{2}\right)}{p^{2}-p_{-} k_{+}+i \varepsilon}\right]\right\} .
\end{aligned}
$$

Carrying out the $k_{-}$contour integration by deforming the contour into the lower half-plane (making the assumption $\left.p_{+}>0\right)$ and using $p_{+} p_{-} \approx p^{2}$, we find that

$$
\begin{aligned}
I_{\mathrm{sub}}\left(p^{2}\right)= & g_{s}^{2} C_{F}\left(\frac{\mu^{2} e^{\gamma_{E}}}{4 \pi}\right)^{\epsilon} \int \frac{d^{D-2} k_{\perp}}{(2 \pi)^{D-2}} \frac{1}{-\boldsymbol{k}_{\perp}^{2}} \\
& \times \int \frac{d k_{+}}{2 \pi}\left\{\left[\frac{\theta\left(p_{+}-k_{+}\right)}{p^{2}-p_{-} k_{+}-\boldsymbol{k}_{\perp}^{2}+i \varepsilon}-\frac{\theta\left(-k_{+}\right)}{p^{2}-p_{-} k_{+}+i \varepsilon}-\frac{\theta\left(k_{+}\right) \theta\left(p_{+}-k_{+}\right)}{p^{2}-p_{-} k_{+}-\frac{p_{+}}{k_{+}} \boldsymbol{k}_{\perp}^{2}+i \varepsilon}\right] p_{-} \bar{J}^{\mathrm{sub}}\left(p^{2}\right)\right. \\
& \left.-\frac{\theta\left(-k_{+}\right)}{k_{+}-i \varepsilon}\left[\bar{J}^{\mathrm{sub}}\left(p^{2}-p_{-} k_{+}\right)-\frac{p^{2} \bar{J}^{\mathrm{sub}}\left(p^{2}\right)}{p^{2}-p_{-} k_{+}+i \varepsilon}\right]\right\} .
\end{aligned}
$$

Rewriting the $\theta$ function in the first term according to $\theta\left(p_{+}-k_{+}\right)=\theta\left(p_{+}-k_{+}\right) \theta\left(k_{+}\right)+\theta\left(-k_{+}\right)$, we arrive at

$$
\begin{aligned}
I_{\text {sub }}\left(p^{2}\right)= & g_{s}^{2} C_{F}\left(\frac{\mu^{2} e^{\gamma_{E}}}{4 \pi}\right)^{\epsilon} \int \frac{d^{D-2} k_{\perp}}{(2 \pi)^{D-2}} \frac{1}{-\boldsymbol{k}_{\perp}^{2}} \int \frac{d k_{+}}{2 \pi}\left\{\theta\left(-k_{+}\right)\left(\frac{1}{p^{2}-p_{-} k_{+}-\boldsymbol{k}_{\perp}^{2}+i \varepsilon}-\frac{1}{p^{2}-p_{-} k_{+}+i \varepsilon}\right) p_{-} \bar{J}^{\mathrm{sub}}\left(p^{2}\right)\right. \\
& +\theta\left(k_{+}\right) \theta\left(p_{+}-k_{+}\right)\left(\frac{1}{p^{2}-p_{-} k_{+}-\boldsymbol{k}_{\perp}^{2}+i \varepsilon}-\frac{1}{p^{2}-p_{-} k_{+}-\frac{p_{+}}{k_{+}} \boldsymbol{k}_{\perp}^{2}+i \varepsilon}\right) p_{-} \bar{J}^{\mathrm{sub}}\left(p^{2}\right) \\
& \left.-\frac{\theta\left(-k_{+}\right)}{k_{+}-i \varepsilon}\left[\bar{J}^{\mathrm{sub}}\left(p^{2}-p_{-} k_{+}\right)-\frac{p^{2} \bar{J}^{\mathrm{sub}}\left(p^{2}\right)}{p^{2}-p_{-} k_{+}+i \varepsilon}\right]\right\} .
\end{aligned}
$$


After carrying out the $k_{\perp}$ integration, we obtain

$$
\begin{aligned}
I_{\mathrm{sub}}\left(p^{2}\right)= & \frac{g_{s}^{2} C_{F}}{4 \pi}\left\{\left(\mu^{2} e^{\gamma_{E}}\right)^{\epsilon} \Gamma\left(\epsilon_{\mathrm{UV}}\right)\left[\int_{-\infty}^{0} \frac{d k_{+}}{2 \pi} \frac{-p_{-}}{\left(-p^{2}+p_{-} k_{+}-i \varepsilon\right)^{1+\epsilon}}+\int_{0}^{p_{+}} \frac{d k_{+}}{2 \pi} \frac{-p_{-}\left(1-\left(\frac{p_{+}}{k_{+}}\right)^{\epsilon}\right)}{\left(-p^{2}+p_{-} k_{+}-i \varepsilon\right)^{1+\epsilon}}\right] \bar{J}^{\mathrm{sub}}\left(p^{2}\right)\right. \\
& \left.+\left(\frac{1}{\epsilon_{\mathrm{UV}}}-\frac{1}{\epsilon_{\mathrm{IR}}}\right) \int_{-\infty}^{0} \frac{d k_{+}}{2 \pi} \frac{1}{k_{+}}\left[\bar{J}^{\mathrm{sub}}\left(p^{2}-p_{-} k_{+}\right)-\frac{p^{2} \bar{J}^{\mathrm{sub}}\left(p^{2}\right)}{p^{2}-p_{-} k_{+}+i \varepsilon}\right]\right\} .
\end{aligned}
$$

Making the change of variables $k_{+}=(1-x) p_{+}$and using $p_{+} p_{-} \approx p^{2}$, we have

$$
\begin{aligned}
I_{\mathrm{sub}}\left(p^{2}\right)= & \frac{\alpha_{s} C_{F}}{2 \pi}\left\{\left(\frac{\mu^{2} e^{\gamma_{E}}}{-p^{2}-i \varepsilon}\right)^{\epsilon} \Gamma\left(\epsilon_{\mathrm{UV}}\right)\left[\int_{1}^{\infty} d x \frac{1}{x^{1+\epsilon}}+\int_{0}^{1} d x \frac{1-(1-x)^{-\epsilon}}{x^{1+\epsilon}}\right] \bar{J}^{\mathrm{sub}}\left(p^{2}\right)\right. \\
& \left.-\left(\frac{1}{\epsilon_{\mathrm{UV}}}-\frac{1}{\epsilon_{\mathrm{IR}}}\right) \int_{1}^{\infty} d x \frac{1}{x(x-1)}\left[x \bar{J}^{\mathrm{sub}}\left(x p^{2}\right)-\bar{J}^{\mathrm{sub}}\left(p^{2}\right)\right]\right\} \\
= & \frac{\alpha_{s} C_{F}}{2 \pi}\left\{\left(\frac{\mu^{2} e^{\gamma_{E}}}{-p^{2}-i \varepsilon}\right)^{\epsilon} \frac{\Gamma\left(-\epsilon_{\mathrm{UV}}\right)^{2} \Gamma(1+\epsilon)}{\Gamma(1-2 \epsilon)} \bar{J}^{\mathrm{sub}}\left(p^{2}\right)-\left(\frac{1}{\epsilon_{\mathrm{UV}}}-\frac{1}{\epsilon_{\mathrm{IR}}}\right) \int_{1}^{\infty} d x \frac{x \bar{J}^{\mathrm{sub}}\left(x p^{2}\right)}{[x(x-1)]_{+}}\right\},
\end{aligned}
$$

where we have used $g_{s}^{2}=4 \pi \alpha_{s}$, and the plus distribution is defined by

$$
\int_{0}^{\infty} d x \frac{f(x)}{[g(x)]_{+}}=\int_{0}^{\infty} \frac{f(x)-f(1)}{[g(x)]_{+}}
$$

Now we can extract the UV divergences of $I_{\text {sub }}\left(p^{2}\right)$ :

$$
I_{\mathrm{sub}}^{\mathrm{UV}}\left(p^{2}\right)=\frac{\alpha_{s} C_{F}}{2 \pi}\left\{\left[\frac{1}{\epsilon_{\mathrm{UV}}^{2}}+\frac{1}{\epsilon_{\mathrm{UV}}} \ln \left(\frac{\mu^{2}}{-p^{2}-i \varepsilon}\right)\right] \bar{J}^{\mathrm{sub}}\left(p^{2}\right)-\frac{1}{\epsilon_{\mathrm{UV}}} \int_{1}^{\infty} d x \frac{x \bar{J}^{\mathrm{sub}}\left(x p^{2}\right)}{[x(x-1)]_{+}}\right\}
$$

Using Eq. (39), we find that the total of the UV divergences in $\bar{J}_{A}^{\text {sub }}\left(p^{2}\right)$ is

$$
\begin{aligned}
\alpha_{s}\left(-Z_{\bar{J}^{\text {sub }}}^{(1)} \otimes \bar{J}^{\mathrm{sub}}\right)_{A} & =\alpha_{s}\left(-Z_{\bar{J}}^{(1)} \otimes \bar{J}^{\mathrm{sub}}\right)_{(\mathrm{a})}+2\left[\alpha_{s}\left(-Z_{\bar{J}}^{(1)} \otimes \bar{J}^{\mathrm{sub}}\right)_{(\mathrm{b})}-\alpha_{s}\left(-Z_{\bar{S}}^{(1)} \otimes \bar{J}^{\mathrm{sub}}\right)_{(\mathrm{a}+\mathrm{b})}\right] \\
& =-\frac{\alpha_{s} C_{F}}{4 \pi} \frac{1}{\epsilon_{\mathrm{UV}}} \bar{J}^{\mathrm{sub}}\left(p^{2}\right)+2\left[I_{\mathrm{b}}^{\mathrm{UV}}\left(p^{2}\right)+I_{\mathrm{sub}}^{\mathrm{UV}}\left(p^{2}\right)\right] \\
& =\frac{\alpha_{s} C_{F}}{4 \pi}\left\{\left[\frac{4}{\epsilon_{\mathrm{UV}}^{2}}+\frac{4}{\epsilon_{\mathrm{UV}}} \ln \left(\frac{\mu^{2}}{-p^{2}-i \varepsilon}\right)+\frac{3}{\epsilon_{\mathrm{UV}}}\right] \bar{J}^{\mathrm{sub}}\left(p^{2}\right)-\frac{4}{\epsilon_{\mathrm{UV}}} \int_{1}^{\infty} d x \frac{x \bar{J}^{\mathrm{sub}}\left(x p^{2}\right)}{[x(x-1)]_{+}}\right\} .
\end{aligned}
$$

\section{B. UV divergences of $\bar{J}_{G}^{\text {sub }}\left(p^{2}\right)$ at order $\alpha_{s}$}

Among the diagrams in Fig. 3, diagrams (e)-(i) are contributions to $\bar{J}_{G}\left(p^{2}\right)$. Only diagrams (e) and (f) give nonvanishing contributions to $\bar{J}_{G}\left(p^{2}\right)$. Diagrams (g) and (h) have the denominators $k^{2}+i \varepsilon, \bar{n} \cdot k+i \varepsilon$, and $(k-\ell)^{2}+i \varepsilon$, which lead to vanishing $k_{+}$contour integrals. This vanishing of the contour integrals is an example of the situation that we mentioned in Sec. III B: These diagrams are not properly part of the radiative jet function because they are not connected to the external photon by lines carrying $n$-hard-collinear momenta and, hence, they do not yield pinch singularities in the $n$-hard-collinear region. Diagrams (i) and (j), which could potentially yield nonlocal renormalizations, have UV-convergent power counts. These are examples of the general argument that we have given in Sec. IV that $\bar{J}$ has only local renormalizations. [Diagrams (i) and (j) also have a vanishing numerator structure in the Feynman gauge.] 
The combined amplitude of diagrams (e) and (f) in Fig. 3 is given by

$$
\begin{aligned}
i \mathcal{A}_{(\mathrm{e}+\mathrm{f})} & =i e_{q} g_{s}^{2} C_{F}\left(\frac{\mu^{2} e^{\gamma_{E}}}{4 \pi}\right)^{\epsilon} \int \frac{d^{D} k}{(2 \pi)^{D}} \frac{\left(\gamma_{\mu}^{\perp}-\frac{\not \not_{\perp} \bar{n}_{\mu}}{\bar{n} \cdot k+i \varepsilon}\right) P_{n}(\not \ell-\not k) \phi_{\perp}^{*}(\not p-\not k) \gamma^{\mu} \not p P_{\bar{n}}}{\left(k^{2}+i \varepsilon\right)\left[(p-k)^{2}+i \varepsilon\right]\left[(\ell-k)^{2}+i \varepsilon\right]\left(p^{2}+i \varepsilon\right)} \\
& =e_{q} g_{s}^{2} C_{F}\left(\frac{\mu^{2} e^{\gamma_{E}}}{4 \pi}\right)^{\epsilon} \phi_{\perp}^{*} \frac{\not h}{2} \frac{i \bar{n} \cdot p}{p^{2}+i \varepsilon} \int \frac{d^{D} k}{(2 \pi)^{D}} \frac{-(D-6) p^{2} \frac{\bar{n} \cdot k}{\bar{n} \cdot p}-4 k \cdot p+2(n \cdot k)(\bar{n} \cdot k)+k^{2}\left(D-6+\frac{2 \bar{n} \cdot p}{\bar{n} \cdot k+i \varepsilon}\right)}{\left(k^{2}+i \varepsilon\right)\left[(p-k)^{2}+i \varepsilon\right]\left[\left(\frac{p^{2}}{2 \bar{n} \cdot p} \bar{n}-k\right)^{2}+i \varepsilon\right]} .
\end{aligned}
$$

We remind the reader that we retain only terms that contribute in leading power in $\lambda$, under the assumption that the momentum $\ell=p-k_{1} \approx \frac{p^{2}}{\bar{n} \cdot p} \frac{\bar{n}}{2}$ is soft and that the momenta $k$ and $k_{1}$ are $n$-hard collinear.

We extract the UV-divergent contribution from the amplitude in Eq. (57) and convolve it with $\bar{J}^{\text {sub }}$ to obtain

$$
\begin{aligned}
\alpha_{s}\left(-Z_{\bar{J}}^{(1)} \otimes \bar{J}^{\text {sub }}\right)_{(\mathrm{e}+\mathrm{f})}= & -i g_{s}^{2} C_{F}\left(\frac{\mu^{2} e^{\gamma_{E}}}{4 \pi}\right)^{\epsilon}\left\{\int \frac{d^{D} k}{(2 \pi)^{D}} \frac{\bar{J}^{\mathrm{sub}}\left(p^{2}\right)}{\left(k^{2}+i \varepsilon\right)\left[(p-k)^{2}+i \varepsilon\right]\left(k^{2}-p_{+} k_{-}+i \varepsilon\right)}\right. \\
& \left.\times\left[-(D-4) p_{+} k_{-}+(D-4) k_{+} k_{-}+(6-D) \boldsymbol{k}_{\perp}^{2}-\frac{2 \boldsymbol{k}_{\perp}^{2} p_{-}}{k_{-}+i \varepsilon}\right]\right\}_{\mathrm{UV}} .
\end{aligned}
$$

For convenience of computation, we split $\left(-Z_{\bar{J}}^{(1)} \otimes \bar{J}^{\text {sub }}\right)_{(\mathrm{e}+\mathrm{f})}$ into two parts, one with the denominator $\frac{1}{k_{-}+i \varepsilon}$ and the other without it:

$$
\begin{aligned}
& \alpha_{s}\left(-Z_{\bar{J}}^{(1)} \otimes \bar{J}^{\mathrm{sub}}\right)_{(\mathrm{e}+\mathrm{f}), 1}=-i g_{s}^{2} C_{F}\left(\frac{\mu^{2} e^{\gamma_{E}}}{4 \pi}\right)^{\epsilon}\left[\int \frac{d^{D} k}{(2 \pi)^{D}} \frac{\left[2 \epsilon p_{+} k_{-}-2 \epsilon k_{+} k_{-}+(2+2 \epsilon) \boldsymbol{k}_{\perp}^{2}\right] \bar{J}^{\mathrm{sub}}\left(p^{2}\right)}{\left(k^{2}+i \varepsilon\right)\left[(p-k)^{2}+i \varepsilon\right]\left(k^{2}-p_{+} k_{-}+i \varepsilon\right)}\right]_{\mathrm{UV}} \\
& \alpha_{s}\left(-Z_{\bar{J}}^{(1)} \otimes \bar{J}^{\mathrm{sub}}\right)_{(\mathrm{e}+\mathrm{f}), 2}=i g_{s}^{2} C_{F}\left(\frac{\mu^{2} e^{\gamma_{E}}}{4 \pi}\right)^{\epsilon}\left[\int \frac{d^{D} k}{(2 \pi)^{D}} \frac{2 \boldsymbol{k}_{\perp}^{2} p_{-} \bar{J}^{\mathrm{sub}}\left(p^{2}\right)}{\left(k_{-}+i \varepsilon\right)\left(k^{2}+i \varepsilon\right)\left[(p-k)^{2}+i \varepsilon\right]\left(k^{2}-p_{+} k_{-}+i \varepsilon\right)}\right]_{\mathrm{UV}}
\end{aligned}
$$

The soft subtractions that correspond to diagrams (e) and (f) of Fig. 3 are given by diagrams (e) and (f) of Fig. 4. Their UV-divergent contributions are

$$
\begin{aligned}
& \alpha_{s}\left(-Z_{\bar{S}}^{(1)} \otimes \bar{J}^{\mathrm{sub}}\right)_{(\mathrm{e}+\mathrm{f})}=-2 i g_{s}^{2} C_{F}\left(\frac{\mu^{2} e^{\gamma_{E}}}{4 \pi}\right)^{\epsilon}\left[\int \frac{d^{D} k}{(2 \pi)^{D}} \frac{P_{n} \not k_{\perp}(\not \ell-\not k) P_{n}\left\{\bar{J}^{\mathrm{sub}}\left(p^{2}\right)-\bar{J}^{\mathrm{sub}}\left[\left(p-k_{+} \frac{\bar{n}}{2}\right)^{2}\right]\right\}}{\left(k^{2}+i \varepsilon\right)\left(k_{+}-i \varepsilon\right)\left(k_{-}+i \varepsilon\right)\left[(\ell-k)^{2}+i \varepsilon\right]}\right]_{\mathrm{UV}} \\
& =i g_{s}^{2} C_{F}\left(\frac{\mu^{2} e^{\gamma_{E}}}{4 \pi}\right)^{\epsilon}\left[\int \frac{d^{D} k}{(2 \pi)^{D}} \frac{-2 k_{\perp}^{2}\left[\bar{J}^{\mathrm{sub}}\left(p^{2}\right)-\bar{J}^{\mathrm{sub}}\left(p^{2}-k_{+} p_{-}\right)\right]}{\left(k^{2}+i \varepsilon\right)\left(k_{-}+i \varepsilon\right)\left(k_{+}-i \varepsilon\right)\left(k^{2}-p_{+} k_{-}+i \varepsilon\right)}\right]_{\mathrm{UV}} \\
& =i g_{S}^{2} C_{F}\left(\frac{\mu^{2} e^{\gamma_{E}}}{4 \pi}\right)^{\epsilon}\left[\int \frac{d^{D} k}{(2 \pi)^{D}} \frac{2 \boldsymbol{k}_{\perp}^{2} p_{-}\left[\bar{J}^{\mathrm{sub}}\left(p^{2}\right)+\frac{p^{2} \bar{J}^{\mathrm{sub}}\left(p^{2}\right)-\left(p^{2}-k_{+} p_{-}\right) \bar{J}^{\mathrm{sub}}\left(p^{2}-k_{+} p_{-}\right)}{-k_{+} p_{-}+i \varepsilon}\right]}{\left(k^{2}+i \varepsilon\right)\left(k_{-}+i \varepsilon\right)\left(p^{2}-k_{+} p_{-}+i \varepsilon\right)\left(k^{2}-p_{+} k_{-}+i \varepsilon\right)}\right]_{\mathrm{UV}} \text {. }
\end{aligned}
$$

Here, we have used Eq. (14) to drop terms that are subleading in the scaling parameter $\lambda$ and have used the facts that $\ell_{+}=p_{+}$and $P_{n} \bar{J}^{\text {sub }}=\bar{J}^{\text {sub }}$. As in our analysis in Sec. VI A we can see that, in the case of the lowest-order expression for $\bar{J}^{\text {sub }}$ in Eq. (35), the expression in Eq. (60) is equal to the soft-approximation of the expression on the right side of Eq. (58). This is the sense in which these soft subtractions correspond to the contributions of diagrams (e) and (f) in Fig. 3. Hence, in the case of the lowest-order expression for $\bar{J}^{\text {sub }}$, the IR divergences in Eq. (60) cancel those in Eq. (58). As we have already mentioned in Sec. VI A, this cancellation does not hold in general because Eq. (58) is missing some of the radiative-jet-function contributions whose IR divergences would be canceled by the soft-subtraction contributions in Eq. (60).

We can also see that, in the case of the lowest-order expression for $\bar{J}^{\text {sub }}$, the nonlocal contributions in Eq. (60) vanish. However, as we have remarked earlier, this feature is special to the lowest-order case.

Owing to the presence of the Wilson-line denominator $k_{-}+i \varepsilon$, the expressions in Eqs. (59b) and (60) develop rapidity divergences as $k_{+} / k_{-} \rightarrow \infty$. These rapidity divergences cancel in the difference between Eqs. (59b) and (60). Therefore, in order to avoid introducing a rapidity regulator, we compute the expressions in Eqs. (59b) and (60) together. We have organized the expression in the third line of Eq. (60) in such a way as to make the cancellation of the rapidity divergences explicit. 
For the jet and soft-subtraction expressions in Eqs. (59) and (60), we perform the $k_{-}$contour integration first. Carrying out the $k_{-}$contour integration by deforming the contour into the lower half-plane (making the assumption $p_{+}>0$ ), we obtain

$$
\begin{aligned}
& \alpha_{S}\left(-Z_{\bar{J}}^{(1)} \otimes \bar{J}^{\mathrm{sub}}\right)_{(\mathrm{e}+\mathrm{f}), 1}=g_{s}^{2} C_{F}\left(\frac{\mu^{2} e^{\gamma_{E}}}{4 \pi}\right)^{\epsilon}\left\{\int \frac{d^{D-2} k_{\perp}}{(2 \pi)^{D-2}} \int_{0}^{p_{+}} \frac{d k_{+}}{2 \pi} \frac{\left(1+\epsilon \frac{p_{+}}{k_{+}}\right) \bar{J}^{\mathrm{sub}}\left(p^{2}\right)}{\left(p^{2}-p_{-} k_{+}-\frac{p_{+}}{k_{+}} \boldsymbol{k}_{\perp}^{2}+i \varepsilon\right) p_{+}}\right\}_{\mathrm{UV}}, \\
& \alpha_{s}\left(-Z_{\bar{J}}^{(1)} \otimes \bar{J}^{\mathrm{sub}}\right)_{(\mathrm{e}+\mathrm{f}), 2}=g_{s}^{2} C_{F}\left(\frac{\mu^{2} e^{\gamma_{E}}}{4 \pi}\right)^{\epsilon}\left\{\int \frac { d ^ { D - 2 } k _ { \perp } } { ( 2 \pi ) ^ { D - 2 } } \left[-\int_{0}^{p_{+}} \frac{d k_{+}}{2 \pi} \frac{p_{-} k_{+}}{\left(p^{2}-p_{-} k_{+}-\frac{p_{+}}{k_{+}} \boldsymbol{k}_{\perp}^{2}+i \varepsilon\right) p_{+} \boldsymbol{k}_{\perp}^{2}}\right.\right. \\
& \left.\left.+\int_{-\infty}^{p_{+}} \frac{d k_{+}}{2 \pi} \frac{p_{-}}{\boldsymbol{k}_{\perp}^{2}\left(p^{2}-p_{-} k_{+}-\boldsymbol{k}_{\perp}^{2}+i \varepsilon\right)}\right] \bar{J}^{\mathrm{sub}}\left(p^{2}\right)\right\}_{\mathrm{UV}}, \\
& \alpha_{S}\left(-Z_{\bar{S}}^{(1)} \otimes \bar{J}^{\mathrm{sub}}\right)_{(\mathrm{e}+\mathrm{f})}=g_{s}^{2} C_{F}\left(\frac{\mu^{2} e^{\gamma_{E}}}{4 \pi}\right)^{\epsilon}\left\{\int \frac{d^{D-2} k_{\perp}}{(2 \pi)^{D-2}} \int_{-\infty}^{p_{+}} \frac{d k_{+}}{2 \pi}\left[-\frac{k_{+} p_{-} \theta\left(k_{+}\right)}{p_{+} \boldsymbol{k}_{\perp}^{2}\left(p^{2}-p_{-} k_{+}+i \varepsilon\right)}+\frac{p_{-}}{\boldsymbol{k}_{\perp}^{2}\left(p^{2}-p_{-} k_{+}+i \varepsilon\right)}\right]\right. \\
& \left.\times\left[\bar{J}^{\mathrm{sub}}\left(p^{2}\right)+\frac{p^{2} \bar{J}^{\mathrm{sub}}\left(p^{2}\right)-\left(p^{2}-k_{+} p_{-}\right) \bar{J}^{\mathrm{sub}}\left(p^{2}-k_{+} p_{-}\right)}{-k_{+} p_{-}+i \varepsilon}\right]\right\}_{\mathrm{UV}} .
\end{aligned}
$$

Note that the expression for $\left(-Z_{\bar{S}}^{(1)} \otimes \bar{J}^{\text {sub }}\right)_{(\mathrm{e}+\mathrm{f})}$ contains a scaleless integral over $k_{\perp}$ that would produce a factor $1 / \epsilon_{\mathrm{UV}}-1 / \epsilon_{\mathrm{IR}}$. That is, the soft subtraction again vanishes in dimensional regularization if one does not distinguish $\mathrm{UV}$ and IR poles, and its role is to convert nonlocal IR poles to nonlocal UV poles.

Using Eq. (39), making the changes of variables $\boldsymbol{k}_{\perp}^{2}=t p^{2}$ and $k_{+}=(1-x) p_{+}$, and using $p_{+} p_{-} \approx p^{2}$, we find that the total contribution to $\left(-Z_{\bar{J}^{\text {sub }}}^{(1)} \otimes \bar{J}^{\text {sub }}\right)_{G}$ is

$$
\begin{aligned}
\alpha_{s}\left(-Z_{\bar{J}^{\text {sub }}}^{(1)} \otimes \bar{J}^{\text {sub }}\right)_{G}= & \alpha_{S}\left[\left(-Z_{\bar{J}}^{(1)} \otimes \bar{J}^{\text {sub }}\right)_{(\mathrm{e}+\mathrm{f}), 1}+\left(-Z_{\bar{J}}^{(1)} \otimes \bar{J}^{\text {sub }}\right)_{(\mathrm{e}+\mathrm{f}), 2}-\left(-Z_{\bar{S}}^{(1)} \otimes \bar{J}^{\mathrm{sub}}\right)_{(\mathrm{e}+\mathrm{f})}\right] \\
= & \frac{\alpha_{s} C_{F}}{2 \pi}\left(\frac{\mu^{2} e^{\gamma_{E}}}{p^{2}}\right)^{\epsilon} \int_{0}^{\infty} \frac{d t t^{-\epsilon}}{\Gamma(1-\epsilon)}\left\{\int_{0}^{1} d x \frac{(-\epsilon-1+x) \bar{J}^{\mathrm{sub}}\left(p^{2}\right)}{t-x(1-x)-i \varepsilon}-\int_{0}^{\infty} d x \frac{1}{t}\left(\frac{1}{t-x-i \varepsilon}+\frac{1}{x}\right) \bar{J}^{\mathrm{sub}}\left(p^{2}\right)\right. \\
& +\int_{0}^{1} d x \frac{1}{t}\left[\frac{(1-x)^{2}}{t-x(1-x)-i \varepsilon}+\frac{1-x}{x}\right] \bar{J}^{\mathrm{sub}}\left(p^{2}\right) \\
& \left.-\int_{0}^{\infty} d x \frac{1}{t x} \frac{\bar{J}^{\mathrm{sub}}\left(p^{2}\right)-x \bar{J}^{\mathrm{sub}}\left(x p^{2}\right)}{x-1}-\int_{0}^{1} d x \frac{1}{t x}\left[\bar{J}^{\mathrm{sub}}\left(p^{2}\right)-x \bar{J}^{\mathrm{sub}}\left(x p^{2}\right)\right]\right\}_{\mathrm{UV}}
\end{aligned}
$$

Completing the $t$ integration, we obtain

$$
\begin{aligned}
& \alpha_{s}\left(-Z_{\bar{J}^{\text {sub }}}^{(1)} \otimes \bar{J}^{\mathrm{sub}}\right)_{G}=\frac{\alpha_{s} C_{F}}{2 \pi}\left\{\left(\frac{\mu^{2} e^{\gamma_{E}}}{-p^{2}-i \varepsilon}\right)^{\epsilon} \Gamma\left(\epsilon_{\mathrm{UV}}\right)\left[\int_{0}^{1} d x \frac{x-1-\epsilon}{[x(1-x)]^{\epsilon}}+\int_{0}^{1} d x \frac{(1-x)^{1-\epsilon}-1}{x^{1+\epsilon}}-\int_{1}^{\infty} \frac{d x}{x^{1+\epsilon}}\right] \bar{J}^{\mathrm{sub}}\left(p^{2}\right)\right. \\
& \left.+\left(\frac{1}{\epsilon_{\mathrm{UV}}}-\frac{1}{\epsilon_{\mathrm{IR}}}\right)\left[\int_{1}^{\infty} d x \frac{x \bar{J}^{\mathrm{sub}}\left(x p^{2}\right)-\bar{J}^{\mathrm{sub}}\left(p^{2}\right)}{x(x-1)}-\int_{0}^{1} d x \frac{x \bar{J}^{\mathrm{sub}}\left(x p^{2}\right)-\bar{J}^{\mathrm{sub}}\left(p^{2}\right)}{1-x}\right]\right\}_{\mathrm{UV}} \\
& =\frac{\alpha_{s} C_{F}}{4 \pi}\left\{\left(\frac{\mu^{2} e^{\gamma_{E}}}{-p^{2}-i \varepsilon}\right)^{\epsilon} \frac{\Gamma(1+\epsilon) \Gamma^{2}\left(-\epsilon_{\mathrm{UV}}\right)}{\Gamma(2-2 \epsilon)}\left(-2+\epsilon-2 \epsilon^{2}\right) \bar{J}^{\mathrm{sub}}\left(p^{2}\right)\right. \\
& \left.+2\left(\frac{1}{\epsilon_{\mathrm{UV}}}-\frac{1}{\epsilon_{\mathrm{IR}}}\right)\left[\int_{1}^{\infty} d x \frac{x \bar{J}^{\mathrm{sub}}\left(x p^{2}\right)}{[x(x-1)]_{+}}-\int_{0}^{1} d x \frac{x \bar{J}^{\mathrm{sub}}\left(x p^{2}\right)}{[1-x]_{+}}\right]\right\}_{\mathrm{UV}}
\end{aligned}
$$

where we have arranged this expression so as to make the cancellation of the rapidity divergences explicit. Finally, extracting the UV divergences, we have 


$$
\begin{aligned}
\alpha_{s}\left(-Z_{\bar{J}^{\mathrm{sub}}}^{(1)} \otimes \bar{J}^{\mathrm{sub}}\right)_{G}= & \frac{\alpha_{s} C_{F}}{4 \pi}\left\{\left[-\frac{2}{\epsilon_{\mathrm{UV}}^{2}}-\frac{2}{\epsilon_{\mathrm{UV}}} \ln \left(\frac{\mu^{2}}{-p^{2}-i \varepsilon}\right)-\frac{3}{\epsilon_{\mathrm{UV}}}\right] \bar{J}^{\mathrm{sub}}\left(p^{2}\right)\right. \\
& \left.+\frac{2}{\epsilon_{\mathrm{UV}}}\left[\int_{1}^{\infty} d x \frac{x \bar{J}^{\mathrm{sub}}\left(x p^{2}\right)}{[x(x-1)]_{+}}-\int_{0}^{1} d x \frac{x \bar{J}^{\mathrm{sub}}\left(x p^{2}\right)}{[1-x]_{+}}\right]\right\} .
\end{aligned}
$$

\section{Renormalization of the subtracted jet function $\bar{J}^{\text {sub }}\left(p^{2}\right)$ at order $\alpha_{s}$}

The total of the UV divergences at order $\alpha_{s}$ is

$$
\begin{aligned}
\alpha_{s}\left(-Z_{\bar{J}^{\text {sub }}}^{(1)} \otimes \bar{J}^{\text {sub }}\right) & =\alpha_{S}\left(-Z_{\bar{J}^{\text {sub }}}^{(1)} \otimes \bar{J}^{\text {sub }}\right)_{A}+\alpha_{S}\left(-Z_{\bar{J}^{\text {sub }}}^{(1)} \otimes \bar{J}^{\text {sub }}\right)_{G} \\
& =\frac{\alpha_{S} C_{F}}{4 \pi}\left\{\left[\frac{2}{\epsilon_{\mathrm{UV}}^{2}}+\frac{2}{\epsilon_{\mathrm{UV}}} \ln \left(\frac{\mu^{2}}{-p^{2}-i \varepsilon}\right)\right] \bar{J}^{\mathrm{sub}}\left(p^{2}\right)-\frac{2}{\epsilon_{\mathrm{UV}}}\left[\int_{1}^{\infty} d x \frac{x \bar{J}^{\mathrm{sub}}\left(x p^{2}\right)}{[x(x-1)]_{+}}+\int_{0}^{1} d x \frac{x \bar{J}^{\mathrm{sub}}\left(x p^{2}\right)}{[1-x]_{+}}\right]\right\} .
\end{aligned}
$$

From Eq. (32b), it then follows that $Z_{J}^{\text {sub }}\left[(1-x), p^{2} ; \mu\right]$, up to order $\alpha_{s}$, is given by

$$
Z_{J}^{\mathrm{sub}}\left[(1-x), p^{2} ; \mu\right]=\left[1+\frac{\alpha_{s} C_{F}}{4 \pi}\left(-\frac{2}{\epsilon^{2}}-\frac{2}{\epsilon} \ln \frac{\mu^{2}}{-p^{2}-i \varepsilon}\right)\right] \delta(1-x)+\frac{\alpha_{s} C_{F}}{2 \pi \epsilon} \Gamma(1, x) \theta(x),
$$

where

$$
\Gamma(1, x)=\left[\frac{\theta(1-x)}{1-x}+\frac{\theta(x-1)}{x(x-1)}\right]_{+} .
$$

This result is in agreement with Eq. (2.6) of Ref. [25] for the case $y=1$. $^{6}$ Therefore, our result for the renormalization kernel of the radiative jet function with soft subtractions agrees with the one-loop result that had been inferred from the factorization theorem for $B \rightarrow \gamma \ell^{-} \nu$ [21]. Note, however, that, in Ref. [21], the renormalization kernel was ascribed to $\bar{J}$, rather than to $\bar{J}^{\text {sub }}$.

The result in Eq. (66) is compatible with the analyticity properties of $\bar{J}$, which is analytic in the $p^{2}$ upper half-plane [22]. Hence, although we have derived this result for the timelike case $p^{2}>0$, it can be continued analytically to the spacelike case $p^{2}<0$. We have also checked the result for the spacelike case by explicit calculation.

\section{VANISHING OF THE SOFT SUBTRACTIONS IN DIMENSIONAL REGULARIZATION}

As we have noted in Secs. VI A and VI B, the order- $\alpha_{s}$ soft subtractions vanish in dimensional regularization if one does not distinguish between UV and IR poles. We now argue that this is a general property of the soft subtractions at all orders in $\alpha_{s}$.

\footnotetext{
${ }^{6}$ Note that, $Z_{J}^{\mathrm{sub}}\left[(1-x), p^{2} ; \mu\right]=Z_{J}^{\mathrm{LN}}\left(p^{2}, x p^{2} ; \mu\right)$, where $Z_{J}^{\mathrm{LN}}\left(p^{2}, x p^{2} ; \mu\right)$ is the quantity that appears in Ref. [25].
}

In the soft subtractions, the only propagator denominators that contain a scale are those that are associated with the $n$-hard-collinear-soft quark line. In these denominators, the scale-dependent terms have the form $k_{i+} p_{-}$, where $k_{i}$ is a loop momentum. If we rescale all of the loop momenta according to

$$
\begin{aligned}
& k_{i-} \rightarrow k_{i-} \rho^{2}, \\
& k_{i \perp} \rightarrow k_{i \perp} \rho, \\
& k_{i+} \rightarrow k_{i+} \rho^{0},
\end{aligned}
$$

then the resulting expression is homogeneous in $\rho$. Therefore, the soft subtractions are scaleless integrals that vanish in dimensional regularization if one does not distinguish between UV and IR divergences. Hence, the soft subtractions do not affect the results of the existing fixedorder calculations of the radiative jet function $[22,25]$.

\section{SUMMARY AND DISCUSSION}

The radiative jet function is a quantity that appears in the factorization theorems for the exclusive processes $B \rightarrow \gamma \ell \nu$ [21] and $H \rightarrow \gamma \gamma$ through a $b$-quark loop [22]. Its renormalization-group evolution is an essential ingredient in the resummation of logarithms of $m_{b} / \mu$ and $m_{H} / m_{b}$ in these processes.

Notwithstanding the importance of these applications, no direct calculation of the renormalization-group kernel for the radiative jet function exists in the literature. Rather, the 
renormalization-group kernel has been inferred from the factorization theorem for $B \rightarrow \gamma \ell \nu$ and the known renormalization-group kernel for the $B$-meson light-front distribution [21].

In this paper, we have argued that the radiative jet function contains, in addition to hard-collinear contributions, soft contributions that must be subtracted in order to avoid double counting of soft contributions that appear in other quantities in the factorization theorems. These soft subtractions are zero-bin subtractions in the language of SCET. We have shown that the radiative jet function can be factored into a convolution over a light-front momentum of a soft-subtraction function and a subtracted radiative jet function. The subtracted radiative jet function is free of soft divergences, which are contained entirely in the softsubtraction function. It is the subtracted jet function that should properly appear in the factorization theorems.

The renormalization-group kernel of the subtracted radiative jet function derives from two sources: (1) the renormalization-group kernel of the radiative jet function, which is local and (2) the renormalization-group kernel of the soft-subtraction function, which is nonlocal and leads to the nonlocal contributions in the renormalization-group kernel of the subtracted radiative jet function.

We have shown that the soft-subtraction contributions contain scaleless integrals in dimensional regularization. That is, they are proportional to $1 / \epsilon_{\mathrm{UV}}-1 / \epsilon_{\mathrm{IR}}$, and they vanish in calculations in which one does not distinguish UV and IR poles. Hence, existing fixed-order calculations of the radiative jet function are not changed by the soft subtractions, which merely convert IR poles to UV poles. This is the usual role of soft (zero-bin) subtractions in factorization theorems. However, because the UV (and IR) divergences that arise from the soft subtractions are nonlocal beyond one-loop order, the soft subtractions give a nonlocal contribution to the renormalization-group kernel of the subtracted radiative jet function. To the best of our knowledge, this is a novel phenomenon.

We have illustrated the role of soft subtractions in the renormalization of the subtracted radiative jet function by carrying out a complete calculation of the renormalizationgroup kernel in order $\alpha_{s}$. Our result agrees with the renormalization-group kernel that was inferred in Ref. [21]. However, in that work, the renormalization-group kernel was ascribed to the radiative jet function, rather than to the subtracted radiative jet function. The renormalizationgroup kernel in order $\alpha_{s}^{2}$ has also been inferred from the factorization theorem for $B \rightarrow \gamma \ell \nu$ [25]. It would be interesting to verify that analysis by making use of the methods that we have presented for the direct calculation of the renormalization-group kernel. However, that work is beyond the scope of the present paper.

There is a large class of exclusive processes that proceed at subleading power in the hard-scattering scale [10]. Helicity-flip processes whose amplitudes contain singularities at the endpoints of light-cone distribution amplitudes are included in this class. While factorization theorems have not yet been worked out in detail for most subleading-power processes, it is known that jet functions are a general feature of the factorization theorems [10]. Since collinear functions, including jet functions at subleading power, generally contain soft contributions that must be subtracted in order to avoid double counting, we expect that our methods would be useful in working out the renormalization/evolution of such jet functions.

In particular, there is a jet function that is identical to the one in Eq. (11), except that the external photon state is replaced by an external gluon state. This jet function would be relevant, for example, in the factorization theorem for $H \rightarrow g g$ through a $b$-quark loop. We would expect the analysis of soft subtractions in the present paper to go through essentially unchanged in this case because softgluon attachments to the external gluon would compensate in the graphical Ward identities for the fact that the external gluon, unlike the external photon, carries color.

The soft function that appears in the factorization theorem for $H \rightarrow \gamma \gamma$ [22,33] also has a nonlocal renormalization kernel. It has been conjectured in Ref. [34] that the complete soft sector in the factorization theorem, which includes contributions from the soft-quark function, two radiative jet functions, and a short-distance Wilson coefficient, is a renormalization-group invariant. That conjecture leads to a prediction for the renormalization-group kernel of the soft function [13], and that prediction was verified in order $\alpha_{s}$ in Ref. [33]. While the soft-subtraction function that we have defined in the present paper clearly has similarities with the soft function in the factorization theorem, it is not obvious that they would combine to cancel the nonlocal contributions to the renormalizationgroup kernel for the complete soft sector. It would be interesting to explore systematically the issue of the renormalization-group invariance of the soft sector by making use of the expression for the subtracted radiative jet function that we have presented in this paper.

\section{ACKNOWLEDGMENTS}

We wish to acknowledge the contributions of Hee Sok Chung to work on related topics at an early stage in this project. We thank Ze Long Liu and Jian Wang for several useful discussions. The work of G. T. B. and X.-P. W. is supported by the U.S. Department of Energy, Division of High Energy Physics, under Contract No. DE-AC0206CH11357. The work of J. L. is supported by the National Research Foundation of Korea under Contract No. NRF-2020R1A2C3009918. The work of J.-H.E. is supported by the National Natural Science Foundation of China (NSFC) through Grant No. 11875112. The submitted manuscript has been created in part by UChicago Argonne, LLC, Operator of Argonne National Laboratory. Argonne, a U.S. Department of Energy Office 
of Science laboratory, is operated under Contract No. DEAC02y-06CH11357. The U.S. Government retains for itself, and others acting on its behalf, a paid-up nonexclusive, irrevocable worldwide license in said article to reproduce, prepare derivative works, distribute copies to the public, and perform publicly and display publicly, by or on behalf of the Government.

All authors contributed equally to this work.

\section{APPENDIX: REARRANGEMENT OF THE BLOB IN THE SECOND DIAGRAM OF FIG. 2}

In this appendix, we sketch, in the diagrammatic approach, the rearrangement of the blob in the second diagram of Fig. 2 into the form of a radiative jet function (with soft subtractions). The issue here is the disposition of gluons that attach to the quark line to the left of the realphoton vertex.

First, we note, as we have mentioned previously, that we have defined $\bar{J}$ in Eq. (11) in such a way that the vertex for a transverse photon is always $i e_{q} \gamma_{\perp}^{\mu}$, regardless of whether the photon attaches to a quark line or to a covariant derivative. Hence, we can treat all real-photon vertices uniformly, regardless of their origin.
Next, we consider an $n$-hard-collinear gluon that connects an $n$-hard-collinear line to the quark line at a point to the left of the real-photon vertex. We write the numerator factor of this gluon as $\gamma \cdot j$, where the Dirac matrix $\gamma$ is on the quark line, and $j$ is the current to which the other end of the gluon attaches. Note that $j$ is the standard QCD current minus the Grammer-Yennie form in the case that there is a soft subtraction associated with the gluon.

We decompose $\gamma \cdot j$ into its light-cone components,

$$
\gamma \cdot j=\frac{1}{2} \gamma \cdot n \bar{n} \cdot j+\frac{1}{2} \gamma \cdot \bar{n} n \cdot j+\gamma_{\perp} \cdot j_{\perp} .
$$

We can further decompose the first term in Eq. (A1) by making use of the identity

$\frac{1}{2} \gamma \cdot n \bar{n} \cdot j=\left(\gamma \cdot k-\gamma_{\perp} \cdot k_{\perp}-\frac{1}{2} \gamma \cdot \bar{n} n \cdot k\right) \frac{\bar{n} \cdot j}{\bar{n} \cdot k}$,

where $k$ is the momentum of the gluon. Rearranging terms, we obtain

$$
\gamma \cdot j=\gamma \cdot k \frac{\bar{n} \cdot j}{\bar{n} \cdot k}+\left(\gamma_{\perp} \cdot j_{\perp}-\gamma_{\perp} \cdot k_{\perp} \frac{\bar{n} \cdot j}{\bar{n} \cdot k}\right)+\frac{1}{2} \gamma \cdot \bar{n} n \cdot j-\frac{1}{2} \gamma \cdot \bar{n} n \cdot k \frac{\bar{n} \cdot j}{\bar{n} \cdot k}
$$

The first term in Eq. (A3) gives the longitudinally polarized (pure gauge) part of the gluon numerator. The terms in parentheses give a transverse-covariant-derivative contribution, where the first term in parentheses is the gauge-field part of the covariant derivative and the second term in parentheses is the ordinary-derivative part of the covariant derivative times a Wilson-line contribution. The last two terms give contributions that are suppressed as $\lambda$ if the lefthand connection of the gluon to the quark line is at the transition on the quark line between a soft momentum and an $n$-hard-collinear momentum.

Suppose that some number of longitudinally polarized gluons attach to the quark line to the left of the realphoton attachment. These can be rearranged by making use of the graphical Ward identities into attachments to a Wilson line $W_{n}^{\dagger}$. If there are no transverse-covariantderivative connections to the quark line to the left of the real-photon attachment, then the Wilson line $W_{n}^{\dagger}$ attaches to the quark line just to the left of the realphoton attachment. Otherwise, the Wilson line $W_{n}^{\dagger}$ attaches to the quark line just to the left of the left-most transverse-covariant-derivative connection. As we have mentioned, at this connection to the quark line, the contributions of the last two terms in Eq. (A3) are suppressed as $\lambda$. However, for connections to the quark line to the right of this connection, the last two terms in Eq. (A3) can contribute in leading power in $\lambda$, and so the complete QCD expression for $\gamma \cdot j$ must be kept for gluons with these connections to the quark line.

Finally, we insert a projector $P_{n}$ to the left of the entire expression to project out the components of the quark field that are large under $n$-hard-collinear scaling. The motivation for this is that we wish to retain only the component of the quark field that corresponds to the effective field theory for the $n$-hard-collinear sector. The presence of $P_{n}$ insures that the blob cannot contain wrong-collinearity contributions.

At this point, the blob in the second diagram of Fig. 2 is in the form of a radiative jet function (with soft subtractions). 
[1] G. T. Bodwin, H. S. Chung, and J. Lee, Double logarithms in $e^{+} e^{-} \rightarrow J / \psi+\eta_{c}$, Phys. Rev. D 90, 074028 (2014).

[2] M. Beneke, G. Buchalla, M. Neubert, and C. T. Sachrajda, QCD factorization for exclusive nonleptonic $B$ meson decays: General arguments and the case of heavy light final states, Nucl. Phys. B591, 313 (2000).

[3] M. Beneke, T. Feldmann, and D. Seidel, Systematic approach to exclusive $B \rightarrow V l^{+} l^{-}, V \gamma$ decays, Nucl. Phys. B612, 25 (2001).

[4] M. Beneke, G. Buchalla, M. Neubert, and C. T. Sachrajda, QCD factorization in $B \rightarrow \pi K, \pi \pi$ decays and extraction of Wolfenstein parameters, Nucl. Phys. B606, 245 (2001).

[5] M. Beneke and T. Feldmann, Factorization of heavy-to-light form factors in soft-collinear effective theory, Nucl. Phys. B685, 249 (2004).

[6] M. Beneke and M. Neubert, QCD factorization for $B \rightarrow P P$ and $B \rightarrow P V$ decays, Nucl. Phys. B675, 333 (2003).

[7] Y. Jia, J. X. Wang, and D. Yang, Bridging light-cone and NRQCD approaches: Asymptotic behavior of $B_{c}$ electromagnetic form factor, J. High Energy Phys. 10 (2011) 105.

[8] M. Benzke, S. J. Lee, M. Neubert, and G. Paz, Factorization at subleading power and irreducible uncertainties in $\bar{B} \rightarrow X_{s} \gamma$ decay, J. High Energy Phys. 08 (2010) 099.

[9] M. Beneke, A. Broggio, S. Jaskiewicz, and L. Vernazza, Threshold factorization of the Drell-Yan process at next-toleading power, J. High Energy Phys. 07 (2020) 078.

[10] I. Moult, I. W. Stewart, and G. Vita, Subleading power factorization with radiative functions, J. High Energy Phys. 11 (2019) 153.

[11] I. Moult, I. W. Stewart, G. Vita, and H. X. Zhu, The soft quark Sudakov, J. High Energy Phys. 05 (2020) 089.

[12] M. van Beekveld, W. Beenakker, E. Laenen, and C. D. White, Next-to-leading power threshold effects for inclusive and exclusive processes with final state jets, J. High Energy Phys. 03 (2020) 106.

[13] C. W. Bauer, S. Fleming, D. Pirjol, and I. W. Stewart, An effective field theory for collinear and soft gluons: Heavy to light decays, Phys. Rev. D 63, 114020 (2001).

[14] C. W. Bauer, D. Pirjol, and I. W. Stewart, Soft-collinear factorization in effective field theory, Phys. Rev. D 65, 054022 (2002).

[15] M. Beneke and T. Feldmann, Multipole-expanded softcollinear effective theory with non-Abelian gauge symmetry, Phys. Lett. B 553, 267 (2003).

[16] C. W. Bauer, S. Fleming, D. Pirjol, I. Z. Rothstein, and I. W. Stewart, Hard scattering factorization from effective field theory, Phys. Rev. D 66, 014017 (2002).

[17] M. Beneke, A. P. Chapovsky, M. Diehl, and T. Feldmann, Soft-collinear effective theory and heavy-to-light currents beyond leading power, Nucl. Phys. B643, 431 (2002).
[18] V. Del Duca, High-energy bremsstrahlung theorems for soft photons, Nucl. Phys. B345, 369 (1990).

[19] D. Bonocore, E. Laenen, L. Magnea, S. Melville, L. Vernazza, and C.D. White, A factorization approach to next-to-leading-power threshold logarithms, J. High Energy Phys. 06 (2015) 008.

[20] D. Bonocore, E. Laenen, L. Magnea, L. Vernazza, and C. D. White, Non-Abelian factorisation for next-to-leading-power threshold logarithms, J. High Energy Phys. 12 (2016) 121.

[21] S. W. Bosch, R. J. Hill, B. O. Lange, and M. Neubert, Factorization and Sudakov resummation in leptonic radiative $B$ decay, Phys. Rev. D 67, 094014 (2003).

[22] Z. L. Liu and M. Neubert, Factorization at subleading power and endpoint-divergent convolutions in $h \rightarrow \gamma \gamma$ decay, J. High Energy Phys. 04 (2020) 033.

[23] Y. M. Wang, Factorization and dispersion relations for radiative leptonic B decay, J. High Energy Phys. 09 (2016) 159.

[24] Y. M. Wang and Y. L. Shen, Subleading-power corrections to the radiative leptonic $B \rightarrow \gamma \ell \nu$ decay in QCD, J. High Energy Phys. 05 (2018) 184.

[25] Z. L. Liu and M. Neubert, Two-loop radiative jet function for exclusive $B$-meson and Higgs decays, J. High Energy Phys. 06 (2020) 060.

[26] J. C. Collins and D. E. Soper, Back-to-back jets in QCD, Nucl. Phys. B193, 381 (1981); B213, 545(E) (1983).

[27] J. Collins, Foundations of perturbative QCD, Cambridge Monographs on Particle Physics, Nuclear Physics and Cosmology, Vol. 32 (Cambridge University Press, Cambridge, England, 2011), pp. 1-624.

[28] A. V. Manohar and I. W. Stewart, The zero-bin and mode factorization in quantum field theory, Phys. Rev. D 76, 074002 (2007).

[29] G. Grammer, Jr. and D. R. Yennie, Improved treatment for the infrared divergence problem in quantum electrodynamics, Phys. Rev. D 8, 4332 (1973).

[30] J. C. Collins, D. E. Soper, and G. F. Sterman, Soft gluons and factorization, Nucl. Phys. B308, 833 (1988).

[31] J. C. Collins, D. E. Soper, and G. F. Sterman, Factorization of hard processes in QCD, Adv. Ser. Dir. High Energy Phys. 5, 1 (1989).

[32] A. V. Manohar, T. Mehen, D. Pirjol, and I. W. Stewart, Reparameterization invariance for collinear operators, Phys. Lett. B 539, 59 (2002).

[33] G. T. Bodwin, J. H. Ee, J. Lee, and X. P. Wang, Analyticity, renormalization, and evolution of the soft-quark function, Phys. Rev. D 104, 016010 (2021).

[34] Z. L. Liu, B. Mecaj, M. Neubert, X. Wang, and S. Fleming, Renormalization and scale evolution of the soft-quark soft function, J. High Energy Phys. 07 (2020) 104. 